\title{
Chinese Medical Injections for Acute Exacerbation of Chronic Obstructive Pulmonary Disease: A Network Meta-analysis
}

\author{
Haiyin $\mathrm{Hu} \mathbb{D}^{\mathrm{I}, *}$ \\ Zhaochen $\mathrm{Ji}^{\mathrm{l}, *}$ \\ Xiaoyu Qiang' \\ Shigang Liu $^{2}$ \\ Xiaodi Sheng' \\ Zhe Chen' \\ Fanqi Liu ${ }^{3}$ \\ Hui Wang' \\ Junhua Zhang'
}

'Evidence-Based Medicine Center, Tianjin University of Traditional Chinese Medicine, Tianjin, People's Republic of China; ${ }^{2}$ Department of Respiratory Diseases, Guang'anmen Hospital, Beijing, People's Republic of China; ${ }^{3}$ Department of Cardiovascular Disease, The Second Affiliated Hospital of Tianjin University of Chinese Medicine, Tianjin, People's Republic of China

*These authors contributed equally to this work
Correspondence: Hui Wang, Junhua

Zhang

Email wanghuil983@foxmail.com;

zjhtcm@foxmail.com
Background: The World Health Organization has indicated that chronic obstructive pulmonary disease (COPD) may become the third leading cause of death by 2030. Acute exacerbation of COPD (AECOPD) is an important process in clinical treatment. Recent studies have shown that Chinese medical injections (CMI) are effective against AECOPD, but the effective difference among different CMIs remains unclear. The aim of this network meta-analysis (NMA) is to compare the therapeutic effect of various CMIs.

Methods: We conducted an overall, systematic literature search in the China National Knowledge Infrastructure, Wanfang, VIP, SinoMed, PubMed, Embase, Cochrane Library, and Web of Science databases to retrieve randomized controlled trials (RCTs) of CMIs for AECOPD published up to January 2021. The Cochrane risk of bias tool was used to assess the risk of bias. Stata 13.1 and WinBUGS 14.3 were used for data analyses.

Results: In total, 103 RCTs involving 8767 participants and 23 CMIs were included. The results indicated that among all treatments conventional Western medical therapy (WM) plus Dengzhanxixin injection (DZXX) led to the best improvement in the clinical efficacy and the ratio of forced expiratory volume in one second $\left(\mathrm{FEV}_{1}\right)$ to forced vital capacity (FVC) (FEV $\left./ \mathrm{FVC}\right)$, with surface under the cumulative ranking curve (SUCRA) $=80.47 \%$ and $98.55 \%$, respectively. Moreover, Shenmai injection (SM) plus WM and Reduning injection (RDN) plus WM led to the best improvement in the $\mathrm{FEV}_{1}(\mathrm{SUCRA}=80.18 \%)$ and the ratio of forced expiratory volume in one second to the predicted value $\left(\mathrm{FEV}_{1} \%, \mathrm{SUCRA}=87.28 \%\right)$. Shengmai injection (SGM) plus WM led to the most considerable shortening in the length of hospital stay (SUCRA=94.70\%). Cluster analysis revealed that $\mathrm{WM}+\mathrm{DZXX}$ had the most favorable response for clinical efficacy and $\mathrm{FEV}_{1}$, as well as clinical efficacy and $\mathrm{FEV}_{1} / \mathrm{FVC}, \mathrm{WM}+\mathrm{RDN}$ had the most favorable response for clinical efficacy and $\mathrm{FEV}_{1} \%, \mathrm{WM}+\mathrm{SGM}$ had the most favorable response for clinical efficacy and length of hospital stay.

Conclusion: $\mathrm{WM}+\mathrm{DZXX}, \mathrm{WM}+\mathrm{RDN}$, and $\mathrm{WM}+\mathrm{SGM}$ were noted to be the optimum treatment regimens for improving in clinical efficacy, $\mathrm{FEV}_{1}, \mathrm{FEV}_{1} / \mathrm{FVC}, \mathrm{FEV}_{1} \%$ and reducing the hospital stay length of AECOPD patients. Considering the limitations this NMA may have, the current results warrant further verification via additional high-quality studies. Keywords: traditional Chinese medicine, TCM, Chinese medical injection, CMI, acute exacerbation of chronic obstructive pulmonary disease, AECOPD, COPD, network metaanalysis, NMA

\section{Introduction}

Chronic obstructive pulmonary disease (COPD) is a lung disease characterized by progressive, persistent airflow restriction and abnormal airway inflammation. When 
the related respiratory symptoms worsen continually, warranting conventional medication changes, the condition is defined as acute exacerbation of COPD (AECOPD). ${ }^{1-3}$ AECOPD can severely impact the patient's daily life and impair their lung function. ${ }^{4}$ According to the World Health Organization, the COPD is expected to be the third leading cause of death by 2030 globally. ${ }^{5-8}$ In the Asia-Pacific region, COPD incidence is estimated to be as high as $6.2 \%$ and rising. ${ }^{9}$ In 2019 an expert consensus on antiinfective therapy for AECOPD in China showed that COPD prevalence in Chinese residents aged $>40$ and $>60$ years was $13.7 \%$ and $>27.0 \% .^{10-12}$ Mortality risk increases significantly in patients with AECOPD. ${ }^{13}$

Corticosteroids and long-acting bronchodilators are recommended as the first-line therapies for AECOPD along with the additional use of antibiotics if required. ${ }^{14}$ However, long-term treatment with systemic corticosteroids is immunosuppressive, which increases the risk and severity of viral infections. ${ }^{15}$ Moreover, the wide application of antibiotics has led to bacterial resistance.$^{16}$ These factors can reduce treatment efficacy further. ${ }^{17}$

Chinese medical injections (CMIs) are widely used in clinical practices. ${ }^{16}$ Some clinical trials have evaluated the efficacy of CMIs for patients with AECOPD and reported their effectiveness in inhibiting inflammation, regulating immune function, and alleviating symptoms. ${ }^{18-20}$

Recent systematic reviews have also shown that CMIs are effective for treating AECOPD, ${ }^{21-25}$ but the effective difference among different CMIs remains unclear. Therefore, in this study, we performed a network metaanalysis (NMA) of all published RCTs on CMIs for treating of AECOPD to compare the therapeutic effect of the different CMIs used.

\section{Methods}

\section{Protocol and Registration}

The study protocol was registered on PROSPERO (Registration No. CRD42021236247; https://www.crd. york.ac.uk/prospero/display_record.php?ID= CRD42021236247).

\section{Eligibility Criteria} Inclusion Criteria

We included RCTs with participants diagnosed with AECOPD (based on diagnosis and treatment guidance of chronic obstructive pulmonary disease). ${ }^{26}$ The experimental group received a CMI plus conventional Western medical therapy (WM) (including oxygen inhalation, spasmolysis, anti-asthmatic and nutritional support, and antibiotic treatment), whereas the control group received WM alone or another CMI plus WM. No restrictions on language, sex, age, and disease course were imposed.

The main outcome was clinical efficacy and the evaluation criteria were as follows:

- Significantly effective: clinical symptoms and signs such as cough and dyspnea disappeared or improved significantly, the pulmonary rales disappeared or decreased, and laboratory examinations showed normal results at the end of the treatment.

- Effective: clinical symptoms, signs, and laboratory examinations, all improved at the end of the treatment.

- Invalid: the condition neither improved nor worsened by the end of the treatment.

Next, clinical efficacy rate was calculated as [(significantly effective cases+effective cases)/total cases] $\times 100 \%$.

The secondary outcomes were as follows:

- Lung function: this included forced expiratory volume in one second $\left(\mathrm{FEV}_{1}\right)$, the ratio of $\mathrm{FEV}_{1}$ to the predicted value $\left(\mathrm{FEV}_{1} \%\right)$, and the ratio of $\mathrm{FEV}_{1}$ to forced vital capacity $\left(\mathrm{FEV}_{1} / \mathrm{FVC}\right)$, as recommended by the Global Strategy for Prevention, Diagnosis and Management of COPD. ${ }^{14}$

- Length of hospital stay: this is closely related to the cost of hospitalization and the economic burden of patients. $^{27}$

The improvements in the lung function and length of hospital stay were expressed as means \pm standard deviations.

\section{Exclusion Criteria}

We excluded studies including AECOPD patients with other comorbidities such as gastroesophageal reflux disease, depression, and osteoporosis - all of which are associated with COPD exacerbation and COPD development acceleration. ${ }^{28}$ We also excluded studies where a combination of multiple TCM injections was used, or where TCM injections were combined with other therapies (decoction, acupuncture, moxibustion, etc). Finally, conference articles, duplicated literature, unavailable studies, and studies with missing data were all excluded. 


\section{Data Sources and Search Strategy}

Eight databases including the China National Knowledge Infrastructure, Wanfang, VIP, SinoMed, PubMed, Embase, Cochrane Library, and Web of Science were searched for eligible studies published from database inception until January 27, 2021. We used search terms including Chinese medicine, injection, COPD, and chronic obstructive pulmonary disease, etc. The complete search strategy is provided in Supplementary File.1. For example, we used the following search strategy on PubMed:

\#1((()((((Pulmonary Disease; Chronic Obstructive [MeSH Terms]) OR (Asthma-Chronic Obstructive Pulmonary Disease Overlap Syndrome[MeSH Terms])) OR (COPD; Severe Early-Onset[Supplementary Concept])) OR (pulmonary disease; chronic obstructive [Title/Abstract])) OR (chronic obstructive pulmonary disease[Title/Abstract])) OR (chronic airflow obstruction [Title/Abstract])) OR (COPD[Title/Abstract])) OR (chronic obstructive lung disease[Title/Abstract])) OR (chronic obstructive airway disease[Title/Abstract])

\#2(((((Traditional Chinese medicine[MeSH Terms]) OR (Traditional Chinese medicine[Text Word])) OR (Chinese medicine[Text Word])) OR (injection[Text Word])) OR (zhongyi[Text Word])) OR (zhongyao[Text Word])

\#3((randomized trials[MeSH Terms]) OR (randomized trials[Text Word])) OR (randomized[Text Word]) \#4\#1 AND \#2 AND \#3

\section{Literature Selection and Data Extraction}

Two researchers independently conducted literature screening and data extraction. Eligible studies were reviewed and the following data were abstracted using a pre-established data extraction table: age, sex, sample size, intervention/ control measures, treatment course, outcomes, and adverse reactions. The selected studies and extracted data were crosschecked by two authors and if there were any disagreements they were resolved through consulting with a third party.

\section{Quality Assessment}

The quality of the included studies was evaluated using the Cochrane risk of bias tool recommended by the Cochrane Handbook for Systematic Reviews Version 5.3. Study quality was evaluated on the basis of seven aspects: random sequence generation, allocation concealment, blinding of participants and personnel, blinding of outcome assessment, incomplete outcome data, selective reporting, and other biases. ${ }^{29}$ For each item the use of the right method was rated as low risk of bias, unclear description was rated as unknown risk of bias, and the use of an incorrect method was rated as high risk of bias. All results were cross-checked by two authors and if there were any disagreements they were resolved through consulting with a third party.

\section{Statistical Analysis}

Dichotomous outcomes were measured as odds ratios (ORs), whereas continuous outcomes were measured as mean differences (MDs). When $95 \%$ confidence interval (CI) of the ORs and MDs did not contain 1 and 0, respectively, the differences were considered statistically significant.

Stata 13.1 was used to draw a network plot-where thicker lines indicated a higher number of the RCTs and a larger dot indicated a larger sample size. An inconsistency test was specifically needed when a closed loop formed in network plot. An inconsistency test was used to mainly evaluate the degree of consistency between the direct comparison results and indirect comparison results. Here $P \geq 0.05$ indicated low inconsistency in the closed loop, whereas $P<0.05$ indicated significant inconsistency.

We used the Markov Chain Monte Carlo method with a random-effect model on WinBUGS 14.3 to perform Bayesian NMA. The iterations were set to 400,000 . The first 100,000 times were used for annealing to eliminate the influence of the initial value and the last 300,000 times were used for sampling. The results are reported as the ORs and MDs with their respective 95\%CIs. Surface under the cumulative ranking curve (SUCRA) was used to rank the efficacy of each intervention. The publication bias was assessed by comparison-adjusted funnel plot with Begg's test. Cluster analysis was conducted using STATA 13.1 to determine the dependency between outcomes and thus to the best interventions. This study was reported in accordance with PRISMA extension for network metaanalysis. $^{30}$

\section{Results}

\section{Literature Search and Characteristics of the Included Studies}

After our preliminary literature search 2430 studies were obtained of which 345 duplicates were removed. A total of 1653 articles were excluded after reading titles and abstracts because they were non-RCTs, non-AECOPD studies, concomitant use of other therapies, included 
patients with other diseases, animal studies, or systematic reviews. Furthermore, 329 studies were excluded after reading full texts because they reported unrelated outcomes, incomplete data, or lack of full text. Finally, 103
RCTs were included. PRISMA flow diagram for study selection is shown in Figure 1.

The characteristics of included studies are shown in Table 1. One hundred and three RCTs comprised a total of

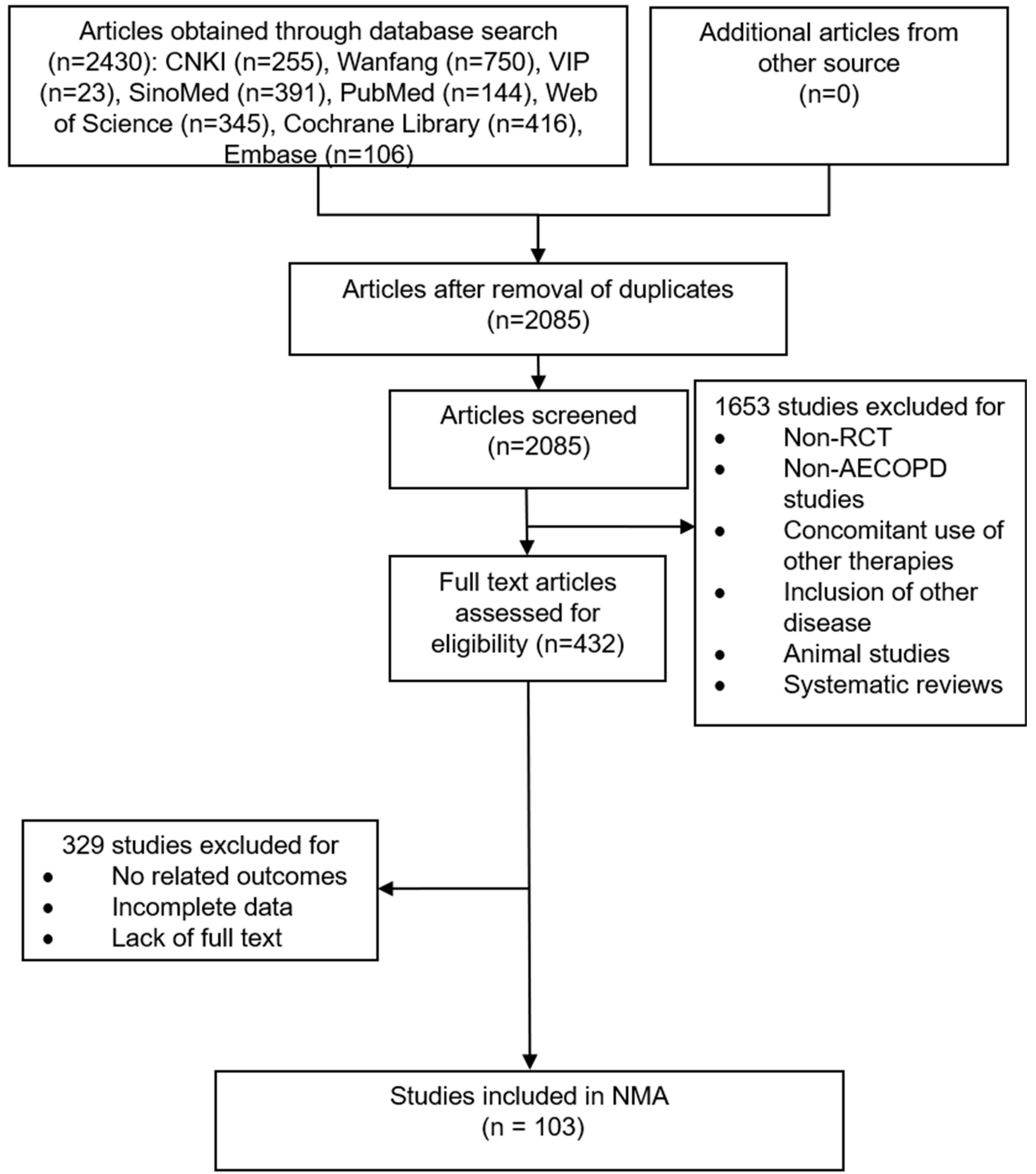

Figure I PRISMA flow diagram.

Notes: PRISMA figure adapted from Hutton B, Salanti G, Caldwell DM, et al.The PRISMA extension statement for reporting of systematic reviews incorporating network meta-analyses of health care interventions: checklist and explanations. Ann Intern Med. 20I5;162(II):777-784. Creative Commons. ${ }^{30}$ 


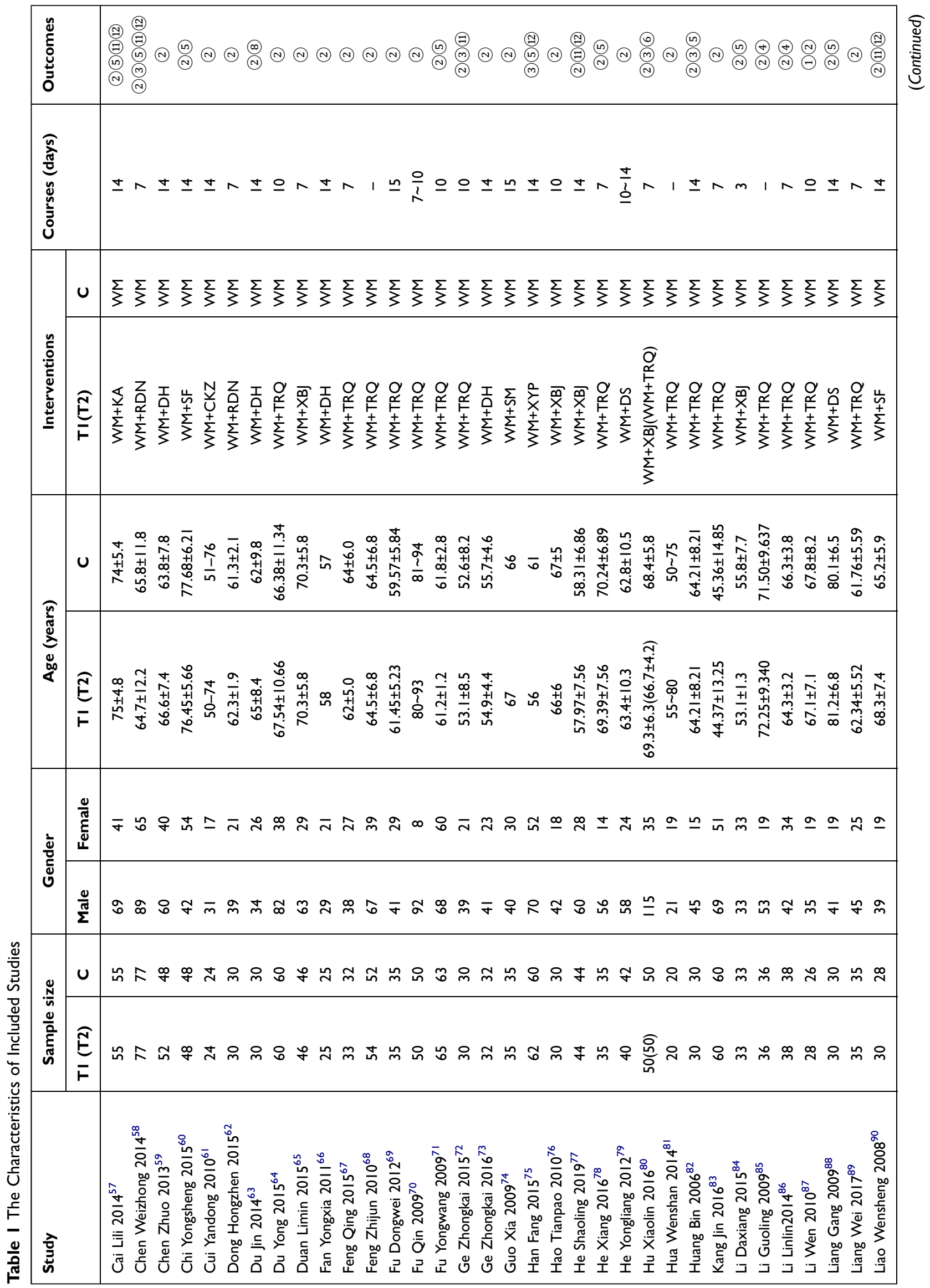




\begin{tabular}{|c|c|c|}
\hline \multicolumn{2}{|c|}{ 嵌 } & (1) \\
\hline \multicolumn{2}{|l|}{ 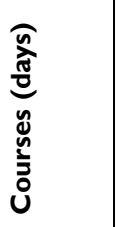 } & $\wedge$ t \\
\hline \multirow[b]{2}{*}{ 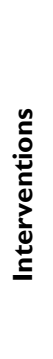 } & $u$ & 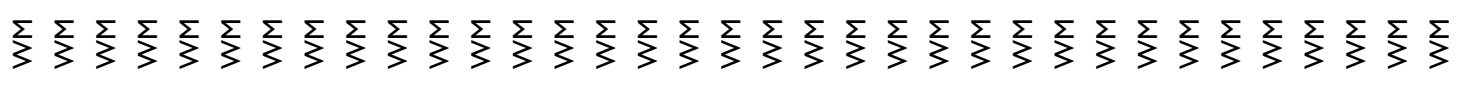 \\
\hline & $\underset{\underline{E}}{\underline{E}}$ & 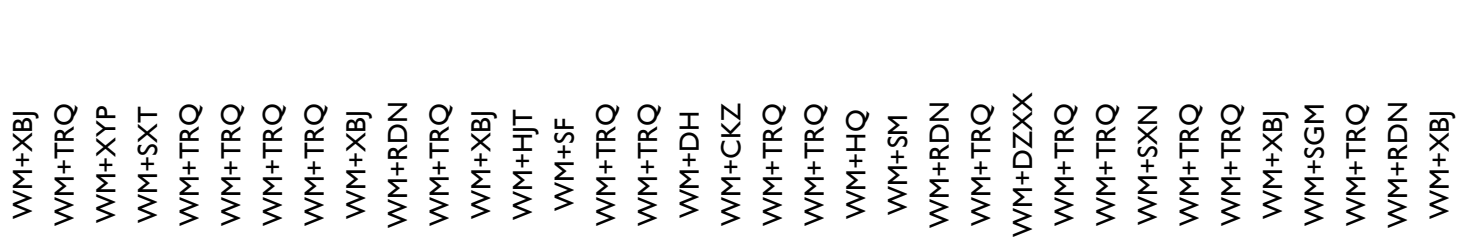 \\
\hline \multirow{2}{*}{ 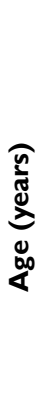 } & $u$ & 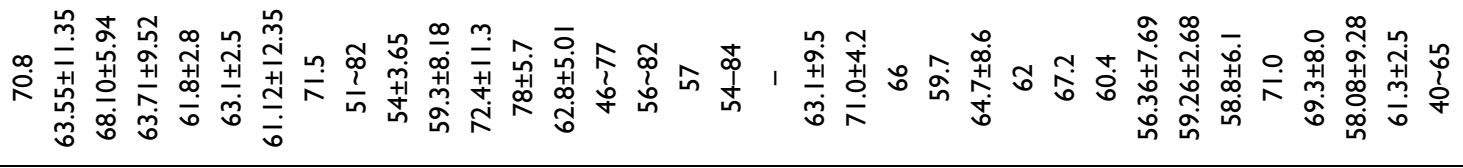 \\
\hline & $\begin{array}{c}\widehat{T} \\
\bar{F}\end{array}$ & 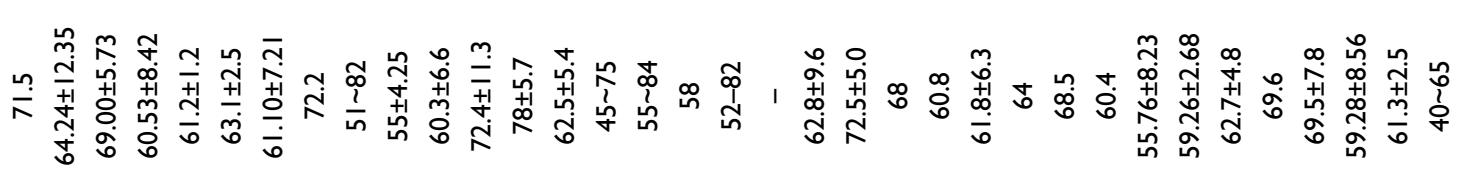 \\
\hline \multirow{2}{*}{ טัँ } & 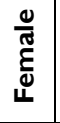 & 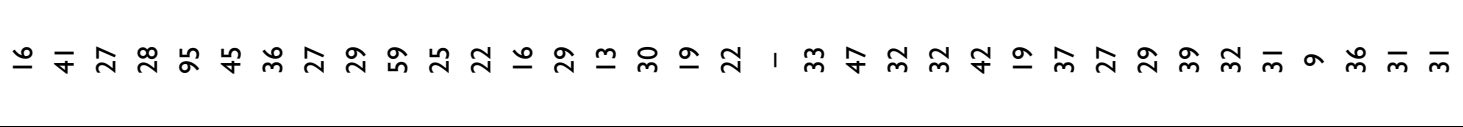 \\
\hline & $\frac{0}{\frac{\pi}{\Sigma}}$ & 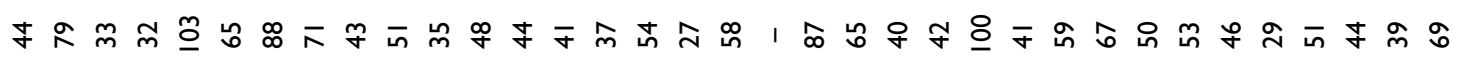 \\
\hline \multirow{2}{*}{$\begin{array}{l}\frac{N}{n} \\
\frac{0}{0} \\
\frac{0}{E} \\
\text { nू }\end{array}$} & $u$ & 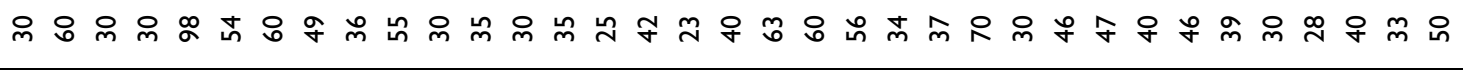 \\
\hline & $\begin{array}{l}\hat{E} \\
\bar{E}\end{array}$ & 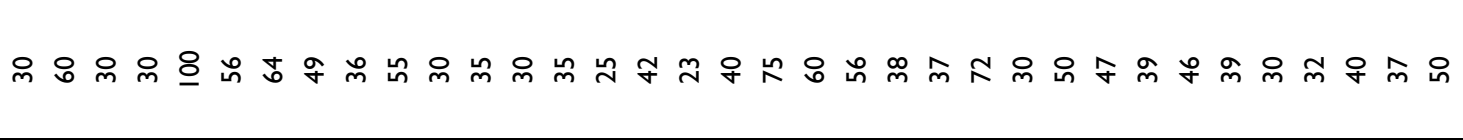 \\
\hline \multicolumn{2}{|l|}{$\begin{array}{l}\text { ते } \\
\text { ڤे }\end{array}$} & 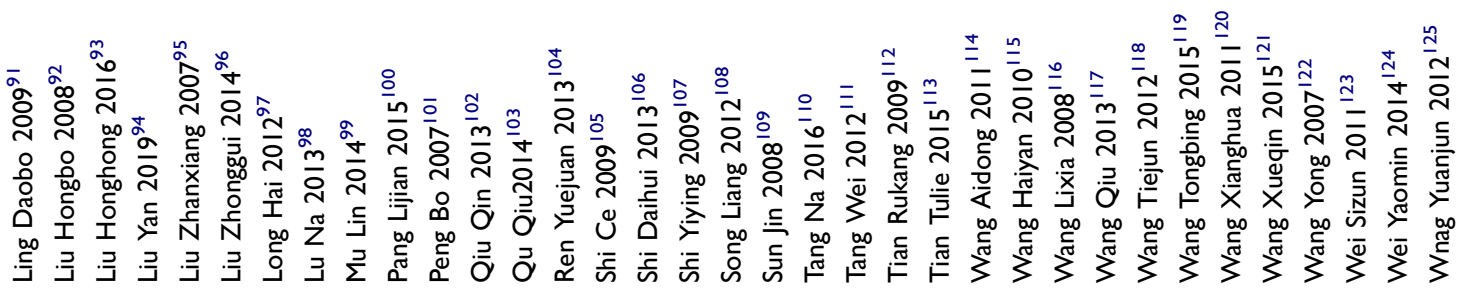 \\
\hline
\end{tabular}




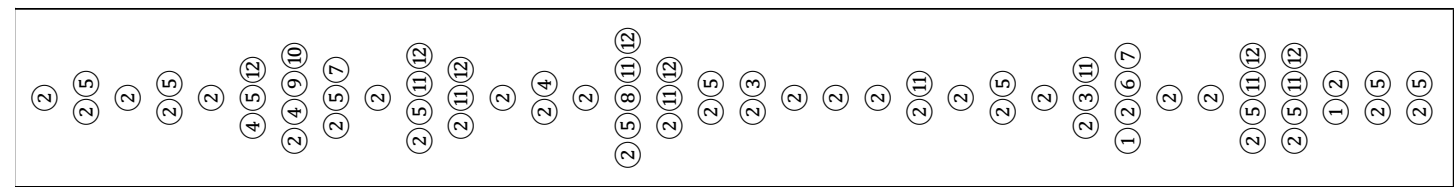

t

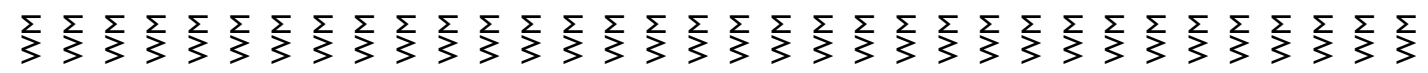

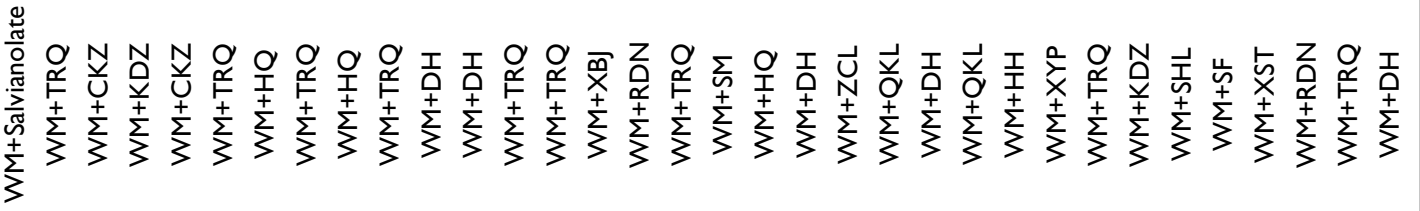

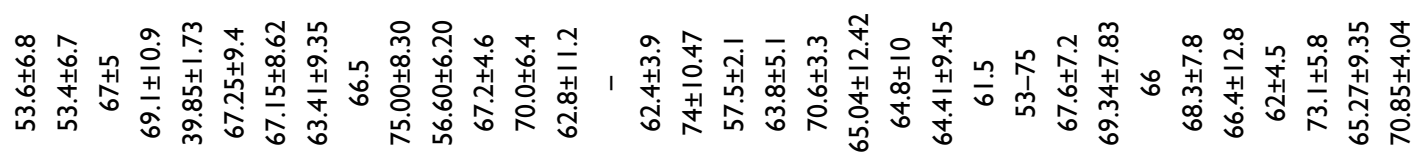

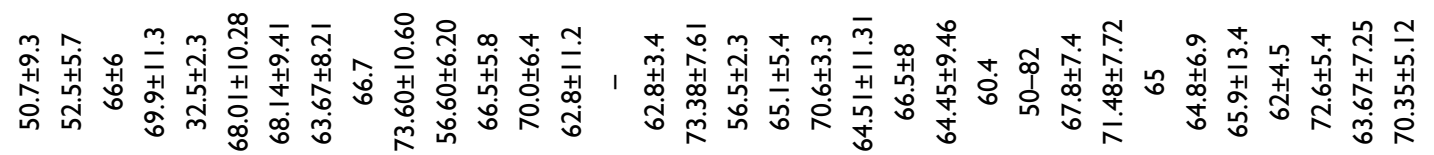

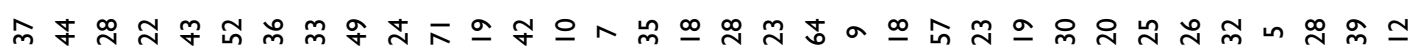

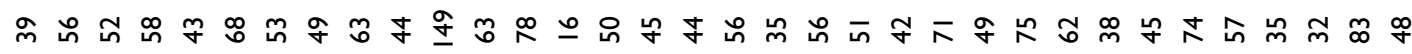

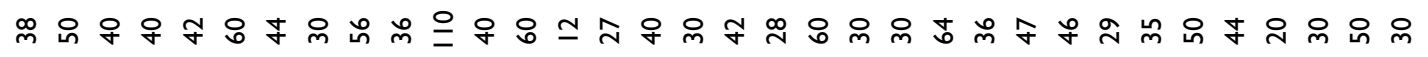

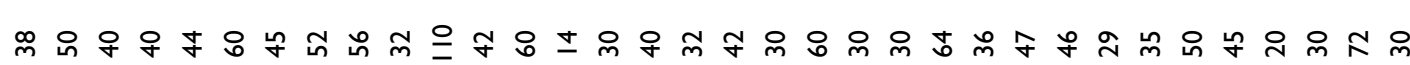

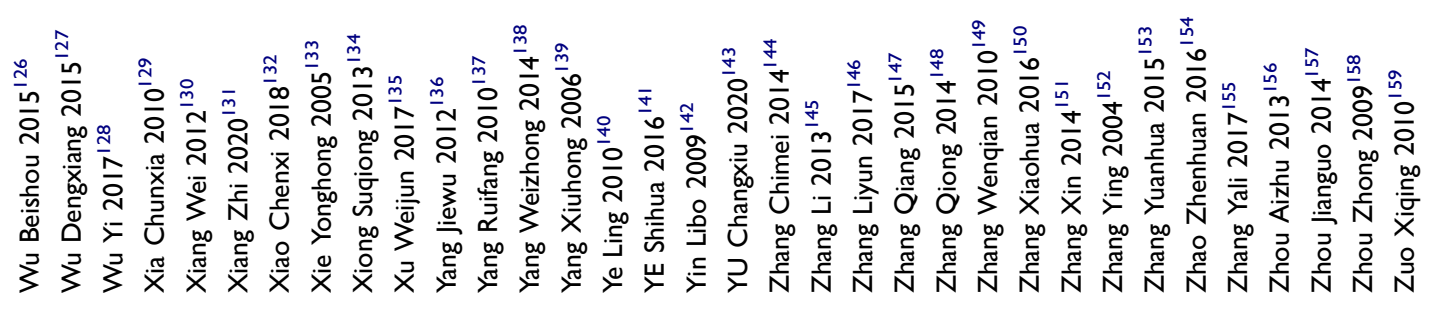


8767 patients, including 4461 and 4306 participants in the treatment group and control group, respectively. The sample size of these studies ranged from 26 to 220. The number of male and female patients was 5502 and 3127 , respectively. However, one study did not report the sex ratio. The participant's ages ranged from 30 to 94 years. All included studies were conducted in China and among them one was a three-arm RCT and 102 were two-arm RCTs.

In total 23 CMIs were included: Chuankezhi injection (CKZ), Danhong injection (DH), Danshen injection (DS), Dengzhanxixin injection (DZXX), Honghua injection (HH), Hongjingtian injection (HJT), Huangqi injection (HQ), Kangai injection (KA), Kudiezi injection (KDZ), Qingkailing injection (QKL), Reduning injection (RDN), Shenfu injection (SF), Shengmai injection (SGM), Shenmai injection (SM), Shuanghuanglian injection (SHL), Shuxuening injection (SXN), Shuxuetong injection (SXT), Tanreqing injection (TRQ), Xiyanping injection (XYP), Xuebijing injection (XBJ), Xuesaitong injection (XST), Zhichuanling injection (ZCL), and Salvianolate injection (Salvianolate). Details about the included CMIs are given in Supplementary Table S1. The treatment duration ranged from 3 to 35 days.

\section{Risk of Bias}

The assessment of risk of bias for all the included studies is illustrated in Figure 2 and Supplementary Table S2.

Regarding random sequence generation, 32 studies used the correct stochastic grouping method and thus were assessed to have low risk, whereas two studies grouped with registration order and thus were assessed to have high risk. The remaining 69 studies reported "random allocation" without specific methods and were assessed to have unclear risk.

Regarding allocation concealment, 102 studies were assessed to have unclear risk because they did not describe their allocation methods. Moreover, one study allotted drugs with a specially assigned person and was assessed to have low risk.

Regarding blinding of participants and personnel, only three studies concealed the used interventions from patients, and thus, these studies were assessed to have low risk. The other 100 studies were assessed to have unclear risk.

Regarding blinding of outcome assessment, 42 studies did not describe the blinding of outcome assessment, but all results assessed using objective indicators, thus, these studies were assessed to have low risk. However, 16 studies used subjective indicators alone to assess result and thus were assessed to have high risk. The remaining studies were deemed to have unclear risk.

Regarding incomplete outcome data, the outcome data of all the included studies were complete, and thus, these studies were assessed to have low risk.

Regarding selective reporting, one study was assessed to have high risk due to the inconformity between its methods and results. The other studies did not report selectively and were assessed to have low risk.

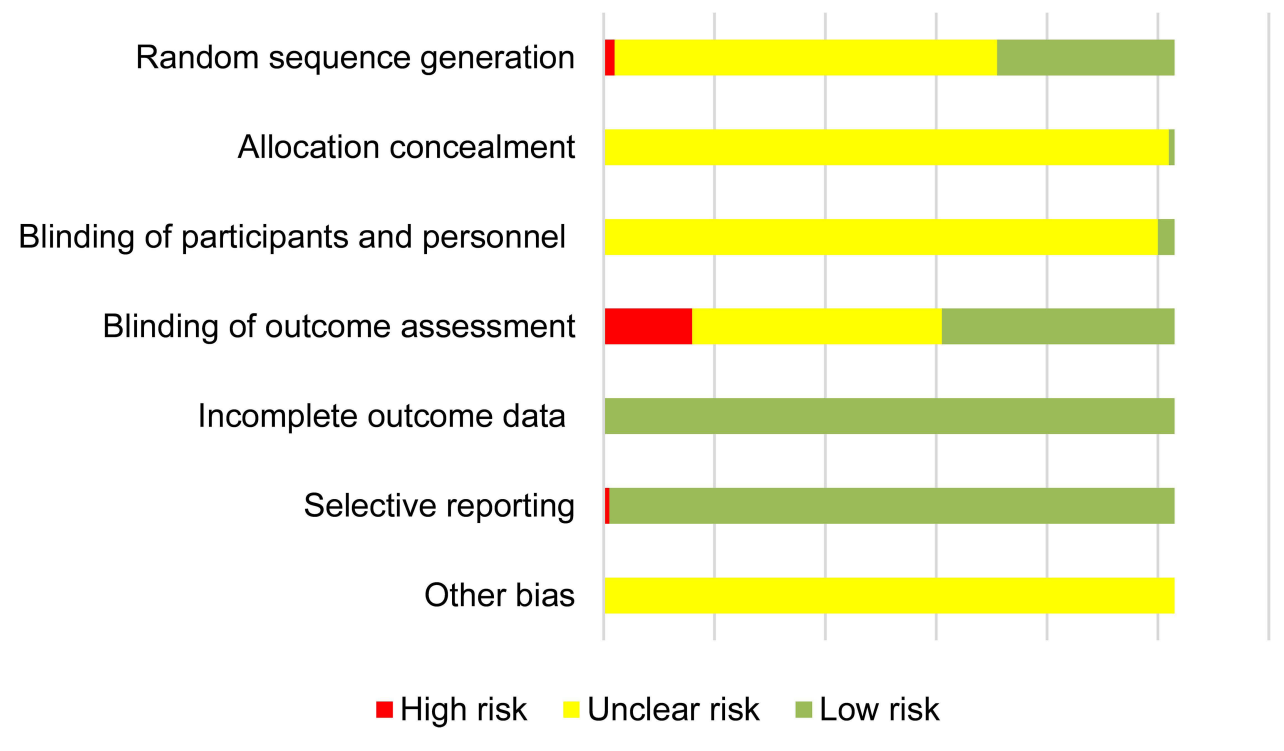

Figure 2 Assessment of the risk of bias. 
All the included studies were deemed to have unclear risk of other bias because some details in these studies (eg, conflict of interest and registration scheme) were unclear.

\section{Bayesian NMA Results Clinical Efficacy}

In total, 99 studies evaluated clinical efficacy, included 22 CMIs and 8326 patients. There are 98 two-arm and one three-arm RCTs, included 23 direct and 230 indirect comparisons. The network plot is presented in Figure 3A.

One closed loop formed in the network plot, and it required an inconsistency test of the direct and indirect comparisons in this closed loop. The results indicated that the inconsistent probability between direct and indirect comparisons in the closed loop WM-(WM+TRQ)-(WM $+\mathrm{XBJ})$ was low $(\mathrm{ROR}=2.261,95 \% \mathrm{CI}: 1.00,6.65, P=0.139$, Supplementary Figure S1 and Figure S2).

The clinical efficacy of $\mathrm{WM}+\mathrm{CKZ}(\mathrm{OR}=5.37,95 \% \mathrm{CI}$ : $1.93,12.25), \mathrm{WM}+\mathrm{DH}(\mathrm{OR}=6.34,95 \% \mathrm{CI}: 3.02,11.96)$, $\mathrm{WM}+\mathrm{DS}(\mathrm{OR}=6.89,95 \% \mathrm{CI}: 1.33,22.74), \mathrm{WM}+\mathrm{DZXX}$ $(\mathrm{OR}=115.4,95 \% \mathrm{CI}: 1.42,514), \mathrm{WM}+\mathrm{KDZ} \quad(\mathrm{OR}=6.88$, 95\%CI: $1.18,24.15), \quad \mathrm{WM}+\mathrm{QKL} \quad(\mathrm{OR}=5.86,95 \% \mathrm{CI}$ : $1.04,20.03), \mathrm{WM}+\mathrm{RDN}(\mathrm{OR}=4.65,95 \% \mathrm{CI}: 2.11,9.08)$, $\mathrm{WM}+\mathrm{SF} \quad(\mathrm{OR}=4.87, \quad 95 \% \mathrm{CI}: \quad 1.59,11.89), \quad \mathrm{WM}+\mathrm{SM}$ $(\mathrm{OR}=4.51,95 \% \mathrm{CI}: 1.14,12.99), \mathrm{WM}+\mathrm{TRQ} \quad(\mathrm{OR}=4.48$,
95\%CI: 3.28,6.02), and $\mathrm{WM}+\mathrm{XBJ}(\mathrm{OR}=3.52,95 \% \mathrm{CI}$ : $1.91,6.1)$ was significantly higher than that of WM alone. Other comparisons did not show significant differences. The detailed results are shown in Table 2.

WM+DZXX was ranked the best in clinical efficacy (SUCRA $=80.47 \%$ ), followed by WM+DH (SUCRA= $66.78 \%$ ) and $\mathrm{WM}+\mathrm{HJT}$ (SUCRA=65.66\%). All SUCRA rankings for clinical efficacy are presented in Supplementary Table S3.

FEV ,

In total, 18 RCTs using eight of the CMIs reported $\mathrm{FEV}_{1}$ improvements in AECOPD patients. The 18 RCTs (one three-arm and 17 two-arm) included 1715 patients. In total, 9 direct and 27 indirect comparisons formed. The network plot is presented in Figure 3B.

In the network plot of the included comparisons that reported $\mathrm{FEV}_{1}$, one closed loop needed an inconsistency test. The direct and indirect comparisons of closed loop $\mathrm{WM}-(\mathrm{WM}+\mathrm{TRQ})-(\mathrm{WM}+\mathrm{XBJ})$ were consistent (ROR=1.004, $95 \% \mathrm{CI}: \quad 1.00, \quad 2.38, \quad P=0.993$, Supplementary Figure S3 and Figure S4).

Of the eight CMIs, only WM+TRQ revealed significant differences in $\mathrm{FEV}_{1}$ compared with $\mathrm{WM}$ alone $(\mathrm{MD}=0.42,95 \% \mathrm{CI}: 0.22,0.62$, Table 3$)$. The network
A

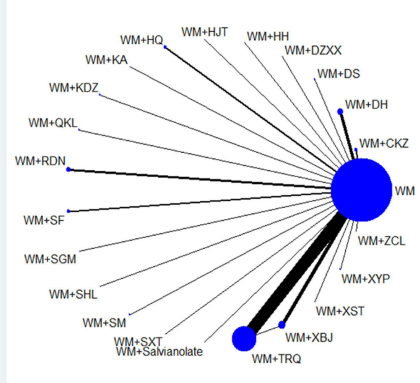

D

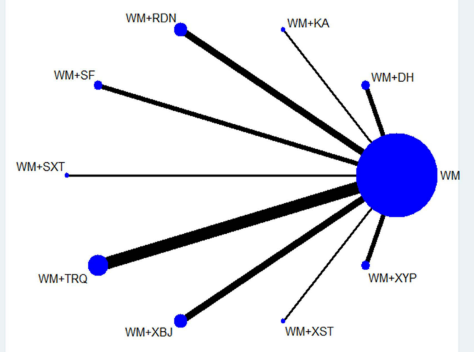

B

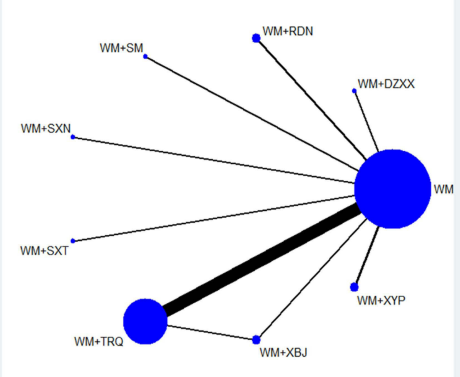

E

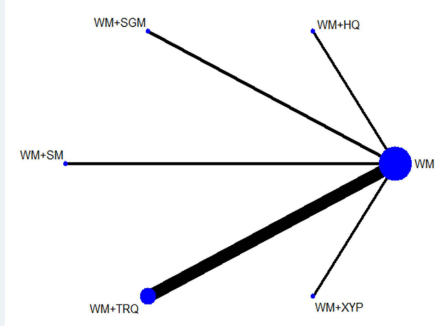

C

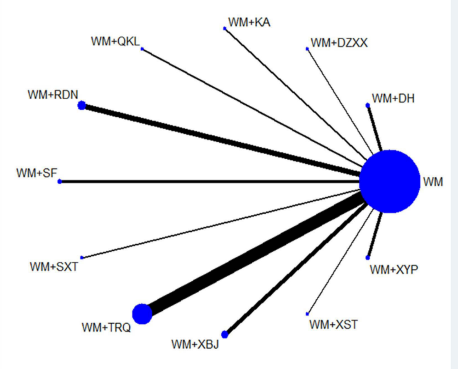

Figure 3 Network plot. (A) Clinical efficacy (B) FEV , (C) FEV /FVC, (D) FEV \%, (E) Length of hospital stay.

Abbreviations: WM, conventional Western medical therapy; CKZ, Chuankezhi injection; DH, Danhong injection; DS, Danshen injection; DZXX, Dengzhanxixin injection; $\mathrm{HH}$, Honghua injection; HJT, Hongjingtian injection; HQ, Huangqi injection; KA, Kangai injection; KDZ, Kudiezi injection; QKL, Qingkailing injection; RDN, Reduning injection; SF, Shenfu injection; SGM, Shengmai injection; SM, Shenmai injection; SHL, Shuanghuanglian injection; SXN, Shuxuening injection; SXT, Shuxuetong injection; TRQ, Tanreqing injection; XYP, Xiyanping injection; XBJ, Xuebijing injection; XST, Xuesaitong injection; ZCL, Zhichuanling injection; Salvianolate, Salvianolate injection. 
Table 2 The Results of Network Meta-analysis of Clinical Efficacy

\begin{tabular}{|c|c|c|c|c|c|c|c|c|c|c|c|}
\hline $\begin{array}{l}\text { OR }(95 \% \\
\mathrm{CI})\end{array}$ & WM & WM+CKZ & WM+DH & WM+DS & $\begin{array}{l}\text { WM } \\
+D Z X X\end{array}$ & WM+HH & WM+HJT & WM+HQ & WM+KA & WM+KDZ & $\begin{array}{l}\text { WM } \\
+Q K L\end{array}$ \\
\hline WM & 1 & & & & & & & & & & \\
\hline$W M+C K Z$ & $\begin{array}{l}5.37(1.93 \\
12.25)^{\mathrm{a}}\end{array}$ & 1 & & & & & & & & & \\
\hline$W M+D H$ & $\begin{array}{l}6.34 \\
(3.02,11.96)^{a}\end{array}$ & $\begin{array}{l}1.47 \\
(0.39,3.91)\end{array}$ & 1 & & & & & & & & \\
\hline WM+DS & $\begin{array}{l}6.89 \\
(1.33,22.74)^{\mathrm{a}}\end{array}$ & $\begin{array}{l}1.6 \\
(0.21,6.11)\end{array}$ & $\begin{array}{l}1.23 \\
(0.19,4.44)\end{array}$ & 1 & & & & & & & \\
\hline$W M+D Z X X$ & $\begin{array}{l}115.4 \\
(1.42,514)^{\mathrm{a}}\end{array}$ & $\begin{array}{l}27.03 \\
(0.25,116.9)\end{array}$ & $\begin{array}{l}20.44 \\
(0.21,92.44)\end{array}$ & $\begin{array}{l}27.07 \\
(0.18,126.2)\end{array}$ & 1 & & & & & & \\
\hline $\mathrm{WM}+\mathrm{HH}$ & $\begin{array}{l}5.25 \\
(0.75,19.02)\end{array}$ & $\begin{array}{l}1.22 \\
(0.12,5.01)\end{array}$ & $\begin{array}{l}0.94 \\
(0.11,3.66)\end{array}$ & $\begin{array}{l}1.29 \\
(0.08,6.02)\end{array}$ & $\begin{array}{l}0.72 \\
(0.01,4.43)\end{array}$ & 1 & & & & & \\
\hline WM+HJT & $\begin{array}{l}15.36 \\
(0.95,77.41)\end{array}$ & $\begin{array}{l}3.59 \\
(0.16,18.94)\end{array}$ & $\begin{array}{l}2.75 \\
(0.14,14.33)\end{array}$ & $\begin{array}{l}3.79 \\
(0.11,21.22)\end{array}$ & $\begin{array}{l}2.15 \\
(0.01,13.97)\end{array}$ & $\begin{array}{l}5.82 \\
(0.14,33.05)\end{array}$ & 1 & & & & \\
\hline $\mathrm{WM}+\mathrm{HQ}$ & $\begin{array}{l}2.21 \\
(0.86,4.74)\end{array}$ & $\begin{array}{l}0.51 \\
(0.12,1.46)\end{array}$ & $\begin{array}{l}0.39 \\
(0.11,1.02)\end{array}$ & $\begin{array}{l}0.54 \\
(0.07,1.95)\end{array}$ & $0.3(0,1.65)$ & $\begin{array}{l}0.83 \\
(0.09,3.35)\end{array}$ & $\begin{array}{l}0.53 \\
(0.02,2.55)\end{array}$ & 1 & & & \\
\hline$W M+K A$ & $\begin{array}{l}14.96 \\
(0.99,73.39)\end{array}$ & $\begin{array}{l}3.5 \\
(0.17,17.86)\end{array}$ & $\begin{array}{l}2.67 \\
(0.15,13.46)\end{array}$ & $\begin{array}{l}3.6 \\
(0.12,20.09)\end{array}$ & $\begin{array}{l}2.02 \\
(0.01,12.95)\end{array}$ & $\begin{array}{l}5.61 \\
(0.15,32.05)\end{array}$ & $\begin{array}{l}3.52 \\
(0.05,21.2)\end{array}$ & $\begin{array}{l}8.16 \\
(0.41,41.85)\end{array}$ & 1 & & \\
\hline$W M+K D Z$ & $\begin{array}{l}6.88 \\
(1.18,24.15)^{a}\end{array}$ & $\begin{array}{l}1.6 \\
(0.19,6.45)\end{array}$ & $\begin{array}{l}1.23 \\
(0.17,4.67)\end{array}$ & $\begin{array}{l}1.69 \\
(0.12,7.76)\end{array}$ & $\begin{array}{l}0.95 \\
(0.01,5.77)\end{array}$ & $\begin{array}{l}2.59 \\
(0.15,12.57)\end{array}$ & $\begin{array}{l}1.65 \\
(0.04,9.18)\end{array}$ & $\begin{array}{l}3.76 \\
(0.46,14.88)\end{array}$ & $\begin{array}{l}1.63 \\
(0.05,8.9)\end{array}$ & 1 & \\
\hline$W M+Q K L$ & $\begin{array}{l}5.86 \\
(1.04,20.03)^{a}\end{array}$ & $\begin{array}{l}1.36 \\
(0.16,5.33)\end{array}$ & $\begin{array}{l}1.05 \\
(0.15,3.9)\end{array}$ & $\begin{array}{l}1.44 \\
(0.11,6.5)\end{array}$ & $\begin{array}{l}0.81 \\
(0.01,4.85)\end{array}$ & $\begin{array}{l}2.21 \\
(0.13,10.72)\end{array}$ & $\begin{array}{l}\text { I.4 } \\
(0.04,7.7 I)\end{array}$ & $\begin{array}{l}3.2 \\
(0.41,12.34)\end{array}$ & $\begin{array}{l}1.39 \\
(0.04,7.5)\end{array}$ & $\begin{array}{l}1.55 \\
(0.1,7.1)\end{array}$ & 1 \\
\hline$W M+R D N$ & $\begin{array}{l}4.65 \\
(2.11,9.08)^{a}\end{array}$ & $\begin{array}{l}1.08 \\
(0.28,2.92)\end{array}$ & $\begin{array}{l}0.83 \\
(0.27,1.99)\end{array}$ & $\begin{array}{l}1.14 \\
(0.16,3.95)\end{array}$ & $\begin{array}{l}0.64 \\
(0.01,3.42)\end{array}$ & $\begin{array}{l}1.75 \\
(0.2,6.88)\end{array}$ & $\begin{array}{l}\text { I.II } \\
(0.05,5.26)\end{array}$ & $\begin{array}{l}2.53 \\
(0.71,6.57)\end{array}$ & $\begin{array}{l}\text { I.I } \\
(0.05,5.04)\end{array}$ & $\begin{array}{l}1.22 \\
(0.15,4.42)\end{array}$ & $\begin{array}{l}\text { I.4I } \\
(0.18,4.98)\end{array}$ \\
\hline $\mathrm{WM}+\mathrm{SF}$ & $\begin{array}{l}4.87 \\
(1.59,11.89)^{\mathrm{a}}\end{array}$ & $\begin{array}{l}1.13 \\
(0.23,3.49)\end{array}$ & $\begin{array}{l}0.87 \\
(0.21,2.46)\end{array}$ & $\begin{array}{l}\text { I.2 } \\
(0.14,4.52)\end{array}$ & $\begin{array}{l}0.67 \\
(0.01,3.78)\end{array}$ & $\begin{array}{l}1.83 \\
(0.17,7.66)\end{array}$ & $\begin{array}{l}\text { I.16 } \\
(0.05,5.7 I)\end{array}$ & $\begin{array}{l}2.66 \\
(0.57,7.99)\end{array}$ & $\begin{array}{l}1.16 \\
(0.05,5.61)\end{array}$ & $\begin{array}{l}1.29 \\
(0.13,5.07)\end{array}$ & $\begin{array}{l}1.47 \\
(0.16,5.7)\end{array}$ \\
\hline WM+SGM & $\begin{array}{l}12.96 \\
(0.76,65.29)\end{array}$ & $3(0.13,16)$ & $\begin{array}{l}2.3 \\
(0.11,12.04)\end{array}$ & $\begin{array}{l}3.19 \\
(0.09,17.75)\end{array}$ & $\begin{array}{l}1.76 \\
(0.01,11.57)\end{array}$ & $\begin{array}{l}4.92 \\
(0.11,28.38)\end{array}$ & $\begin{array}{l}3.15 \\
(0.04,19.09)\end{array}$ & $\begin{array}{l}7.01 \\
(0.32,37.55)\end{array}$ & $\begin{array}{l}3.15 \\
(0.04,18.65)\end{array}$ & $\begin{array}{l}3.37 \\
(0.09,19.29)\end{array}$ & $\begin{array}{l}3.88 \\
(0.11,22.07)\end{array}$ \\
\hline$W M+S M$ & $\begin{array}{l}4.51 \\
(1.14,12.99)^{\mathrm{a}}\end{array}$ & $\begin{array}{l}1.05 \\
(0.17,3.64)\end{array}$ & $\begin{array}{l}0.81 \\
(0.16,2.58)\end{array}$ & $\begin{array}{l}\text { I.II } \\
(0.11,4.54)\end{array}$ & $\begin{array}{l}0.62 \\
(0.01,3.58)\end{array}$ & $\begin{array}{l}\text { I.7I } \\
(0.13,7.64)\end{array}$ & $\begin{array}{l}\text { I.08 } \\
(0.04,5.59)\end{array}$ & $\begin{array}{l}2.46 \\
(0.43,8.31)\end{array}$ & $\begin{array}{l}1.07 \\
(0.04,5.42)\end{array}$ & $\begin{array}{l}1.19 \\
(0.1,5.01)\end{array}$ & $\begin{array}{l}1.37 \\
(0.12,5.73)\end{array}$ \\
\hline $\mathrm{WM}+\mathrm{SHL}$ & $\begin{array}{l}12.92 \\
(0.85,64.72)\end{array}$ & $\begin{array}{l}3 \\
(0.14,15.91)\end{array}$ & $\begin{array}{l}2.3 \\
(0.13,11.9)\end{array}$ & $\begin{array}{l}3.17 \\
(0.1,17.66)\end{array}$ & $\begin{array}{l}1.78 \\
(0.01,11.62)\end{array}$ & $\begin{array}{l}4.87 \\
(0.13,28.5)\end{array}$ & $\begin{array}{l}3.1 \\
(0.04,18.82)\end{array}$ & $\begin{array}{l}7.05 \\
(0.35,36.75)\end{array}$ & $\begin{array}{l}3.11 \\
(0.04,18.92)\end{array}$ & $\begin{array}{l}3.42 \\
(0.1,19.23)\end{array}$ & $\begin{array}{l}3.91 \\
(0.12,22.12)\end{array}$ \\
\hline $\mathrm{WM}+\mathrm{SXT}$ & $\begin{array}{l}8.69 \\
(0.5,44.36)\end{array}$ & $\begin{array}{l}2.04 \\
(0.08,10.84)\end{array}$ & $\begin{array}{l}1.55 \\
(0.07,8.18)\end{array}$ & $\begin{array}{l}2.15 \\
(0.06,12.23)\end{array}$ & $\begin{array}{l}1.21 \\
(0,7.84)\end{array}$ & $\begin{array}{l}3.33 \\
(0.08,19.25)\end{array}$ & $\begin{array}{l}2.09 \\
(0.02,12.94)\end{array}$ & $\begin{array}{l}4.73 \\
(0.21,25.34)\end{array}$ & $\begin{array}{l}2.08 \\
(0.03,12.69)\end{array}$ & $\begin{array}{l}2.3 \\
(0.06,13.14)\end{array}$ & $\begin{array}{l}2.63 \\
(0.07,15.04)\end{array}$ \\
\hline$W M+T R Q$ & $\begin{array}{l}4.48 \\
(3.28,6.02)^{\mathrm{a}}\end{array}$ & $\begin{array}{l}I .04 \\
(0.34,2.4 I)\end{array}$ & $\begin{array}{l}0.8 \\
(0.35,1.56)\end{array}$ & $\begin{array}{l}\text { I.I } \\
(0.19,3.46)\end{array}$ & $\begin{array}{l}0.62 \\
(0.01,3.19)\end{array}$ & $\begin{array}{l}1.69 \\
(0.23,6.1)\end{array}$ & $\begin{array}{l}1.07 \\
(0.06,4.8)\end{array}$ & $\begin{array}{l}2.45 \\
(0.89,5.39)\end{array}$ & $\begin{array}{l}1.06 \\
(0.06,4.59)\end{array}$ & $\begin{array}{l}1.18 \\
(0.18,3.9)\end{array}$ & $\begin{array}{l}1.35 \\
(0.21,4.4)\end{array}$ \\
\hline$W M+X Y P$ & $\begin{array}{l}2.93 \\
(0.64,8.76)\end{array}$ & $\begin{array}{l}0.68 \\
(0.1,2.42)\end{array}$ & $\begin{array}{l}0.52 \\
(0.09,1.73)\end{array}$ & $\begin{array}{l}0.72 \\
(0.06,3)\end{array}$ & $0.4(0,2.38)$ & $\begin{array}{l}\text { I.I } \\
(0.08,5.04)\end{array}$ & $\begin{array}{l}0.7 \\
(0.02,3.7)\end{array}$ & $\begin{array}{l}\text { I.6 } \\
(0.25,5.49)\end{array}$ & $\begin{array}{l}0.69 \\
(0.02,3.53)\end{array}$ & $\begin{array}{l}0.77 \\
(0.06,3.34)\end{array}$ & $\begin{array}{l}0.88 \\
(0.07,3.77)\end{array}$ \\
\hline$W M+X B J$ & $\begin{array}{l}3.52 \\
(1.91,6.1)^{\mathrm{a}}\end{array}$ & $\begin{array}{l}0.82 \\
(0.24,2.08)\end{array}$ & $\begin{array}{l}0.63 \\
(0.23,1.4)\end{array}$ & $\begin{array}{l}0.86 \\
(0.13,2.88)\end{array}$ & $\begin{array}{l}0.48 \\
(0.01,2.56)\end{array}$ & $\begin{array}{l}1.33 \\
(0.16,5.04)\end{array}$ & $\begin{array}{l}0.84 \\
(0.04,3.9)\end{array}$ & $\begin{array}{l}1.92 \\
(0.6,4.69)\end{array}$ & $\begin{array}{l}0.83 \\
(0.04,3.74)\end{array}$ & $\begin{array}{l}0.93 \\
(0.13,3.22)\end{array}$ & $\begin{array}{l}1.06 \\
(0.15,3.64)\end{array}$ \\
\hline WM+XST & $\begin{array}{l}82.43 \\
(0.5228 .6)\end{array}$ & $\begin{array}{l}17.99 \\
(0.09,53.05)\end{array}$ & $\begin{array}{l}15.58 \\
(0.08,40.28)\end{array}$ & $\begin{array}{l}17.17 \\
(0.06,55.81)\end{array}$ & $\begin{array}{l}6.33 \\
(0.01,30.04)\end{array}$ & $\begin{array}{l}59.16 \\
(0.08,85.58)\end{array}$ & $\begin{array}{l}21.27 \\
(0.03,52.97)\end{array}$ & $\begin{array}{l}41.88 \\
(0.22,125.1)\end{array}$ & $\begin{array}{l}17.74 \\
(0.03,52.69)\end{array}$ & $\begin{array}{l}22.32 \\
(0.06,59.95)\end{array}$ & $\begin{array}{l}22.45 \\
(0.08,69.31)\end{array}$ \\
\hline$W M+Z C L$ & $\begin{array}{l}7.36 \\
(0.38,37.47)\end{array}$ & $\begin{array}{l}\text { I.7I } \\
(0.06,9.09)\end{array}$ & $\begin{array}{l}\text { I.3I } \\
(0.06,6.91)\end{array}$ & $\begin{array}{l}1.8 \\
(0.05,10.11)\end{array}$ & $\begin{array}{l}1.01 \\
(0,6.5 I)\end{array}$ & $\begin{array}{l}2.77 \\
(0.06,15.95)\end{array}$ & $\begin{array}{l}1.74 \\
(0.02,10.62)\end{array}$ & $\begin{array}{l}4 \\
(0.16,21.4)\end{array}$ & $\begin{array}{l}1.76 \\
(0.02,10.63)\end{array}$ & $\begin{array}{l}1.95 \\
(0.05,11.19)\end{array}$ & $\begin{array}{l}2.24 \\
(0.05,12.62)\end{array}$ \\
\hline
\end{tabular}




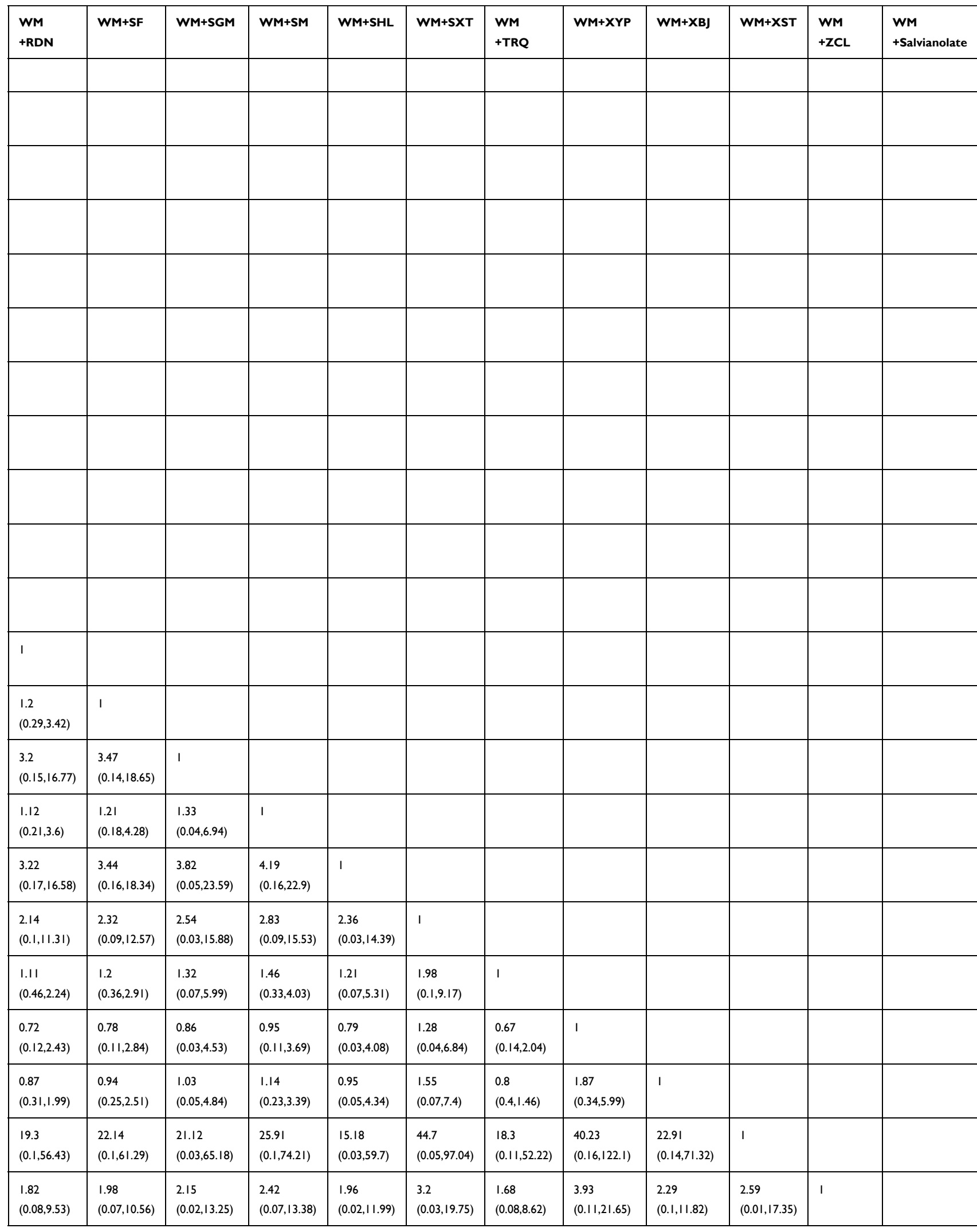

(Continued) 
Table 2 (Continued).

\begin{tabular}{|c|c|c|c|c|c|c|c|c|c|c|c|}
\hline $\begin{array}{l}\text { OR }(95 \% \\
\mathrm{CI})\end{array}$ & WM & $W M+C K Z$ & WM+DH & WM+DS & $\begin{array}{l}\text { WM } \\
+D Z X X\end{array}$ & WM+HH & WM+HJT & WM+HQ & WM+KA & WM+KDZ & $\begin{array}{l}\text { WM } \\
+Q K L\end{array}$ \\
\hline $\begin{array}{l}\text { WM } \\
+ \text { Salvianolate }\end{array}$ & $\begin{array}{l}2.69 \\
(0.36,10.05)\end{array}$ & $\begin{array}{l}0.63 \\
(0.06,2.64)\end{array}$ & $\begin{array}{l}0.48 \\
(0.05,1.93)\end{array}$ & $\begin{array}{l}0.66 \\
(0.04,3.14)\end{array}$ & $\begin{array}{l}0.37 \\
(0,2.28)\end{array}$ & $\begin{array}{l}\text { I.02 } \\
(0.05,5.14)\end{array}$ & $\begin{array}{l}0.65 \\
(0.01,3.63)\end{array}$ & $\begin{array}{l}\text { I. } 47 \\
(0.14,6.06)\end{array}$ & $\begin{array}{l}0.63 \\
(0.02,3.51)\end{array}$ & $\begin{array}{l}0.71 \\
(0.04,3.45)\end{array}$ & $\begin{array}{l}0.82 \\
(0.04,3.95)\end{array}$ \\
\hline
\end{tabular}

Note: ${ }^{\text {a The }} 95 \% \mathrm{Cls}$ of the ORs did not contain I.

Abbreviations: OR, odds ratio; Cl, confidence interval; WM, conventional Western medical therapy; CKZ, Chuankezhi injection; DH, Danhong injection; DS, Danshen injection; DZXX, Dengzhanxixin injection; HH, Honghua injection; HJT, Hongjingtian injection; HQ, Huangqi injection; KA, Kangai injection; KDZ, Kudiezi injection; QKL, Qingkailing injection; RDN, Reduning injection; SF, Shenfu injection; SGM, Shengmai injection; SM, Shenmai injection; SHL, Shuanghuanglian injection; SXT, Shuxuetong injection; TRQ, Tanreqing injection; XYP, Xiyanping injection; XBJ, Xuebijing injection; XST, Xuesaitong injection; ZCL, Zhichuanling injection; Salvianolate, Salvianolate injection.

Table 3 The Results of Network Meta-analysis of FEV,

\begin{tabular}{|c|c|c|c|c|c|c|c|c|c|}
\hline $\begin{array}{l}\text { MD } \\
(95 \% \mathrm{Cl})\end{array}$ & WM & $\begin{array}{l}\text { WM } \\
+D Z X X\end{array}$ & WM+RDN & WM+SM & WM+SXN & WM+SXT & WM+TRQ & WM+XYP & $\begin{array}{l}\text { WM } \\
+X B J\end{array}$ \\
\hline WM & 0 & & & & & & & & \\
\hline $\begin{array}{l}\text { WM } \\
+ \text { DZXX }\end{array}$ & $0.33(-0.34, I)$ & 0 & & & & & & & \\
\hline $\begin{array}{l}\text { WM } \\
+ \text { RDN }\end{array}$ & $\begin{array}{l}0.31(-0.12, \\
0.75)\end{array}$ & $\begin{array}{l}-0.02(-0.82, \\
0.78)\end{array}$ & 0 & & & & & & \\
\hline$W M+S M$ & $0.59(0,1.18)$ & $\begin{array}{l}0.26(-0.63, \\
\text { I.15) }\end{array}$ & $\begin{array}{l}0.28(-0.46, \\
\text { I.01) }\end{array}$ & 0 & & & & & \\
\hline $\begin{array}{l}\text { WM } \\
+S X N\end{array}$ & $\begin{array}{l}0.38(-0.21, \\
0.97)\end{array}$ & $\begin{array}{l}0.05(-0.84 \\
0.94)\end{array}$ & $\begin{array}{l}0.07(-0.67, \\
0.8)\end{array}$ & $\begin{array}{l}-0.21(-1.05 \\
0.63)\end{array}$ & 0 & & & & \\
\hline $\begin{array}{l}\text { WM } \\
+S X T\end{array}$ & $\begin{array}{l}0.21(-0.39, \\
0.81)\end{array}$ & $\begin{array}{l}-0.12(-1.02, \\
0.78)\end{array}$ & $\begin{array}{l}-0.1(-0.84 \\
0.64)\end{array}$ & $\begin{array}{l}-0.38(-1.22, \\
0.46)\end{array}$ & $\begin{array}{l}-0.17(-1.01, \\
0.67)\end{array}$ & 0 & & & \\
\hline $\begin{array}{l}\text { WM } \\
+T R Q\end{array}$ & $\begin{array}{l}0.42(0.22 \\
0.62)^{\mathrm{a}}\end{array}$ & $\begin{array}{l}0.09(-0.61, \\
0.79)\end{array}$ & $\begin{array}{l}0.11(-0.37 \\
0.58)\end{array}$ & $\begin{array}{l}-0.17(-0.8 \\
0.45)\end{array}$ & $\begin{array}{l}0.04(-0.59 \\
0.66)\end{array}$ & $\begin{array}{l}0.21(-0.42 \\
0.84)\end{array}$ & 0 & & \\
\hline $\begin{array}{l}\text { WM } \\
+X Y P\end{array}$ & $\begin{array}{l}0.25(-0.17 \\
0.67)\end{array}$ & $\begin{array}{l}-0.08(-0.87, \\
0.7 I)\end{array}$ & $\begin{array}{l}-0.06(-0.67, \\
0.54)\end{array}$ & $\begin{array}{l}-0.34(-1.07 \\
0.39)\end{array}$ & $\begin{array}{l}-0.13(-0.86, \\
0.6)\end{array}$ & $\begin{array}{l}0.04(-0.69 \\
0.77)\end{array}$ & $\begin{array}{l}-0.17(-0.64, \\
0.3)\end{array}$ & 0 & \\
\hline$W M+X B J$ & $\begin{array}{l}0.3(-0.25 \\
0.85)\end{array}$ & $\begin{array}{l}-0.03(-0.9, \\
0.83)\end{array}$ & $\begin{array}{l}-0.01(-0.71, \\
0.69)\end{array}$ & $\begin{array}{l}-0.29(-1.1, \\
0.52)\end{array}$ & $\begin{array}{l}-0.08(-0.89, \\
0.73)\end{array}$ & $\begin{array}{l}0.09(-0.72, \\
0.9)\end{array}$ & $\begin{array}{l}-0.12(-0.67, \\
0.44)\end{array}$ & $\begin{array}{l}0.05(-0.65, \\
0.75)\end{array}$ & 0 \\
\hline
\end{tabular}

Note: ${ }^{\text {a The }} 95 \% \mathrm{Cls}$ of the MDs did not contain 0 .

Abbreviations: MD, mean difference; $\mathrm{Cl}$, confidence interval; WM, conventional Western medical therapy; DZXX, Dengzhanxixin injection; RDN, Reduning injection; SM, Shenmai injection; SXN, Shuxuening injection; SXT, Shuxuetong injection; TRQ, Tanreqing injection; XYP, Xiyanping injection; XBJ, Xuebijing injection.

analysis showed no significant differences between other comparisons.

In the probability rankings, $\mathrm{WM}+\mathrm{SM}(\mathrm{SUCRA}=80.18 \%)$ was the most likely to improve $\mathrm{FEV}_{1}$ in the patients with AECOPD, followed by WM+TRQ (SUCRA $=66.73 \%$ ), WM + SXN (SUCRA=58.81\%). SUCRA rankings for $\mathrm{FEV}_{1}$ are presented in Supplementary Table S3.

\section{$\mathrm{FEV}_{1} / \mathrm{FVC}$}

In 27 two-arm RCTs, the changes in $\mathrm{FEV}_{1} / \mathrm{FVC}$ before and after treatment with 11 CMIs plus WM in 2362 patients with AECOPD were examined. This led to 11 direct and 55 indirect comparisons forming. The network plot for $\mathrm{FEV}_{1} / \mathrm{FVC}$ is shown in Figure 3C.

Of the 11 CMIs, WM+DZXX (MD=19.25, 95\%CI: 9.15, 29.32), $\mathrm{WM}+\mathrm{KA} \quad(\mathrm{MD}=8.14,95 \% \mathrm{CI}: 0.24,16.05)$, WM $+\mathrm{RDN}(\mathrm{MD}=8.8,95 \% \mathrm{CI}: 4.41,13.28)$, and $\mathrm{WM}+\mathrm{TRQ}$ $(\mathrm{MD}=6.54,95 \% \mathrm{CI}: 3.84,9.27)$ were more effective than $\mathrm{WM}$ alone in improving the $\mathrm{FEV}_{1} / \mathrm{FVC}$ in patients with AECOPD. Moreover, $\mathrm{WM}+\mathrm{DH}$ was inferior to $\mathrm{WM}+\mathrm{DZXX}$ $(\mathrm{MD}=17.96,95 \% \mathrm{CI}: 6.52,29.43)$ and $\mathrm{WM}+\mathrm{RDN}(\mathrm{MD}=7.52$, $95 \%$ CI: $0.52,14.57$ ), whereas $\mathrm{WM}+\mathrm{DZXX}$ was superior to 


\begin{tabular}{|c|c|c|c|c|c|c|c|c|c|c|c|}
\hline $\begin{array}{l}\text { WM } \\
+R D N\end{array}$ & WM+SF & WM+SGM & WM+SM & WM+SHL & WM+SXT & $\begin{array}{l}\text { WM } \\
+T R Q\end{array}$ & WM+XYP & WM+XBJ & WM+XST & $\begin{array}{l}\mathrm{WM} \\
+\mathrm{ZCL}\end{array}$ & $\begin{array}{l}\text { WM } \\
\text { +Salvianolate }\end{array}$ \\
\hline $\begin{array}{l}0.66 \\
(0.07,2.69)\end{array}$ & $\begin{array}{l}0.72 \\
(0.06,3.1)\end{array}$ & $\begin{array}{l}0.8 \\
(0.02,4.54)\end{array}$ & $\begin{array}{l}0.88 \\
(0.06,3.94)\end{array}$ & $\begin{array}{l}0.73 \\
(0.02,4.07)\end{array}$ & $\begin{array}{l}1.19 \\
(0.03,6.74)\end{array}$ & $\begin{array}{l}0.61 \\
(0.08,2.32)\end{array}$ & $\begin{array}{l}1.44 \\
(0.09,6.7)\end{array}$ & $\begin{array}{l}0.83 \\
(0.09,3.26)\end{array}$ & $\begin{array}{l}0.99 \\
(0.01,6.26)\end{array}$ & $\begin{array}{l}1.53 \\
(0.03,8.85)\end{array}$ & 1 \\
\hline
\end{tabular}

$\mathrm{WM}+\mathrm{QKL}(\mathrm{MD}=-16.17,95 \% \mathrm{CI}:-29.36,-2.95), \mathrm{WM}+\mathrm{SF}$ $(\mathrm{MD}=-15.35,95 \% \mathrm{CI}:-27,-3.7), \mathrm{WM}+\mathrm{SXT}(\mathrm{MD}=-16.51$, 95\%CI: $-29.45,-3.55)$, WM+TRQ (MD $=-12.71,95 \% \mathrm{CI}$ : $-23.11,-2.25$ ), $\mathrm{WM}+\mathrm{XYP}(\mathrm{MD}=-16.39,95 \% \mathrm{CI}:-28.05$, $-4.71)$, and $\mathrm{WM}+\mathrm{XBJ}(\mathrm{MD}=-14.8,95 \% \mathrm{CI}:-26.04,-3.52)$. The detailed results are shown in Table 4. Other comparisons did not reach statistical significance.

$\mathrm{WM}+\mathrm{DZXX}$ was ranked the best in $\mathrm{FEV}_{1} / \mathrm{FVC}$ (SUCRA $=98.55 \%$ ), followed by $\mathrm{WM}+\mathrm{RDN}$ (SUCRA $=77.15 \%$ ) and WM+KA (SUCRA=69.47\%). SUCRA rankings for $\mathrm{FEV}_{1} / \mathrm{FVC}$ are presented in Supplementary Table S3.

\section{$\mathrm{FEV}, \%$}

$\mathrm{FEV}_{1} \%$ was reported in 20 two-arm RCTs, including nine CMIs and 1838 patients - forming 9 direct and 36 indirect comparisons. The network plot for $\mathrm{FEV}_{1} \%$ is shown in Figure 3D.

$\mathrm{WM}+\mathrm{RDN} \quad(\mathrm{MD}=11.5,95 \% \mathrm{CI}: 6.57,16.41), \mathrm{WM}$ + TRQ $\quad(\mathrm{MD}=6.64, \quad 95 \% \mathrm{CI}: 2.58, \quad 10.64), \quad \mathrm{WM}+\mathrm{XYP}$ $(\mathrm{MD}=9.12,95 \% \mathrm{CI}: 2.57,15.56), \mathrm{WM}+\mathrm{XBJ}(\mathrm{MD}=8.39$, $95 \%$ CI: $2.91,13.79)$ were significantly superior to $\mathrm{WM}$ alone in increasing $\mathrm{FEV}_{1} \%$. Other comparisons did not show significant results. The detailed results are shown in Table 5.

For $\mathrm{FEV}_{1} \%$, WM+RDN (SUCRA=87.28\%) was ranked the best, followed by WM+XYP (SUCRA=69.75\%), WM $+\mathrm{XBJ}$ (SUCRA=64.86\%). SUCRA rankings for $\mathrm{FEV}_{1} \%$ are presented in Supplementary Table S3.

\section{Length of Hospital Stay}

Eight two-arm RCTs including five CMIs and 717 patients recorded the length of hospital stay and formed 5 direct and 10 indirect comparisons. The network plot for length of hospital stay is shown in Figure 3E.

$\mathrm{WM}+\mathrm{SGM}(\mathrm{MD}=-6.9,95 \% \mathrm{CI}:-10.89,-2.9)$ and WM+TRQ (MD $=-3.07,95 \% \mathrm{CI}:-4.97,-1.15$ ) led to a shorter length of hospital stay than did WM alone
(Table 6). There was no significant difference in other comparisons.

In terms of shortening the length of hospital stay, WM+SGM (SUCRA=94.70\%) was ranked the best, followed by $\mathrm{WM}+\mathrm{SM}(\mathrm{SUCRA}=57.49 \%)$ and $\mathrm{WM}+\mathrm{XYP}$ (SUCRA $=55.85 \%$ ). SUCRA rankings for length of hospital stay are presented in Supplementary Table S3.

\section{Cluster Analysis Plot for Outcomes}

Cluster analysis was performed on clinical efficacy and $\mathrm{FEV}_{1}$, clinical efficacy and $\mathrm{FEV}_{1} / \mathrm{FVC}$, clinical efficacy and $\mathrm{FEV}_{1} \%$, clinical efficacy and length of hospital stay so as to find the best interventions. The results showed that the most favorable response by WM+DZXX were for clinical efficacy and $\mathrm{FEV}_{1}$ as well as for clinical efficacy and $\mathrm{FEV}_{1} / \mathrm{FVC}$, by $\mathrm{WM}+\mathrm{RDN}$ were for clinical efficacy and $\mathrm{FEV}_{1} \%$, and by $\mathrm{WM}+\mathrm{SGM}$ were for clinical efficacy and length of hospital stay (Figure 4).

\section{Publication Bias}

Begg's test was used to identify the possible publication bias related to the different interventions and the impact of small sample studies. The results demonstrated potential publication bias in the funnel plot of clinical efficacy ( $P=0.000)$, suggesting that the publication bias was small in the funnel plot for $\mathrm{FEV}_{1}(P=0.347), \mathrm{FEV}_{1} / \mathrm{FVC}$ $(P=0.359)$, and $\mathrm{FEV}_{1} \%(P=0.381$, Supplementary Figure S5). Because the number of included studies that reported the length of hospital stay was $<10$, we did not assess the publication bias for length of hospital stay.

\section{Safety}

Of the 103 included RCTs, 49 reported regarding adverse reactions, of these 49 studies, 29 reported that no adverse reactions appeared. The remaining 20 studies included 1539 patients and $12 \mathrm{TCM}$ injections. Of the 742 patients who received WM, $50(6.74 \%)$ had the following adverse 


\begin{tabular}{|c|c|c|c|c|c|c|c|c|c|c|c|c|}
\hline$\sum \begin{array}{l}5 \\
\vdots \\
+\end{array}$ & & & & & & & & & & & & $\circ$ \\
\hline $\begin{array}{l}\bar{x} \\
x \\
\frac{+}{z} \\
3\end{array}$ & & & & & & & & & & & 0 & 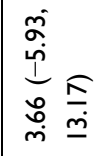 \\
\hline$\sum_{\substack{\Sigma \\
x}}^{0}$ & & & & & & & & & & 。 & 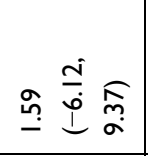 & 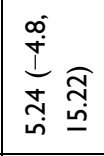 \\
\hline 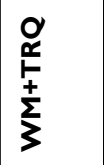 & & & & & & & & & 0 & 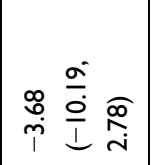 & 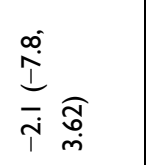 & 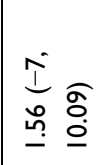 \\
\hline $\begin{array}{l}5 \\
x \\
\sum^{+} \\
\sum_{3}^{1}\end{array}$ & & & & & & & & 0 & 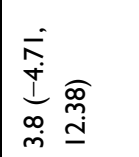 & 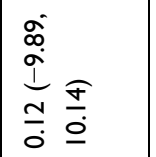 & 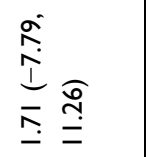 & 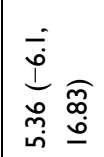 \\
\hline $\begin{array}{l}\frac{\Delta}{0} \\
\frac{1}{2} \\
3\end{array}$ & & & & & & & 0 & 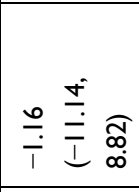 & 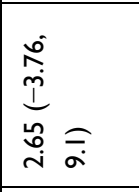 & 兽 & 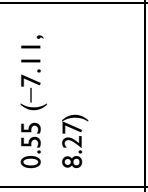 & 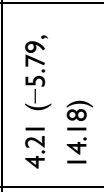 \\
\hline 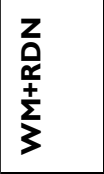 & & & & & & 0 & 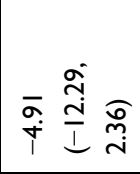 & îj & 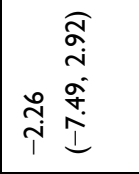 & 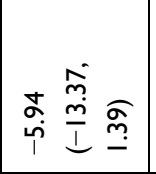 & 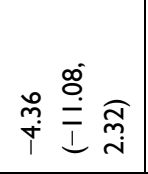 & 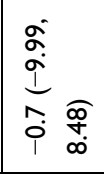 \\
\hline $\begin{array}{l}\overrightarrow{1} \\
\stackrel{y}{0} \\
\stackrel{+}{\sum} \\
3\end{array}$ & & & & & 0 & 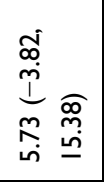 & 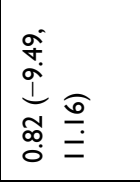 & 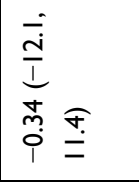 & 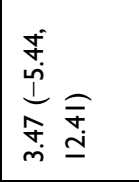 & 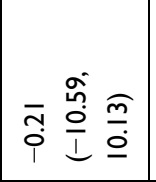 & 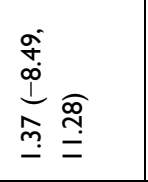 & 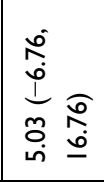 \\
\hline 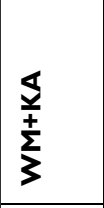 & & & & 0 & 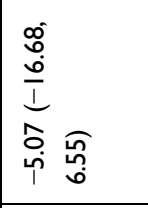 & $\begin{array}{l}0 \\
0 \\
\infty \\
0 \\
0 \\
0 \\
0 \\
0 \\
0\end{array}$ & 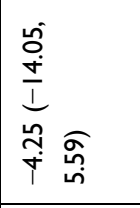 & 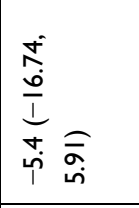 & 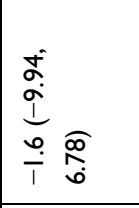 & 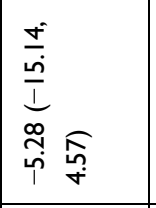 & 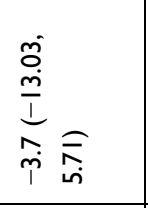 & \\
\hline 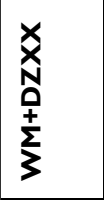 & & & $\circ$ & 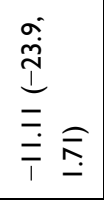 & 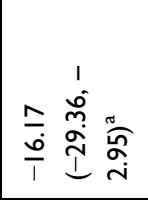 & 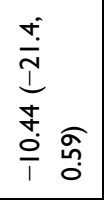 & 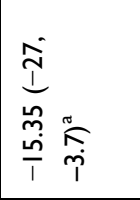 & 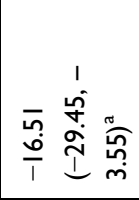 & 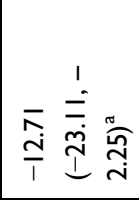 & 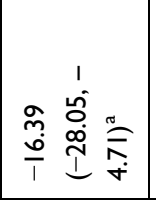 & 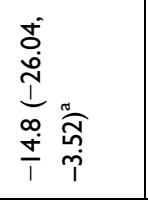 & 疍 \\
\hline $\begin{array}{l}\frac{1}{2} \\
\sum_{3}^{+} \\
3\end{array}$ & & 0 & 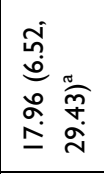 & 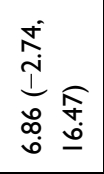 & 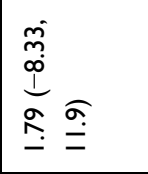 & 罂 & 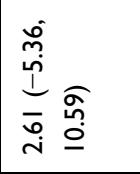 & 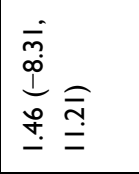 & 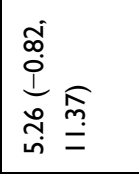 & 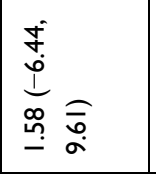 & 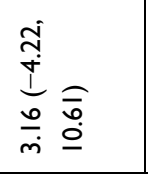 & 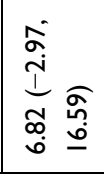 \\
\hline$\sum$ & 0 & 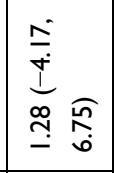 & 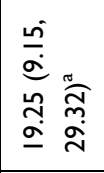 & 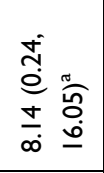 & 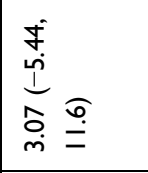 & 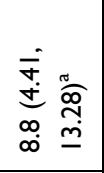 & 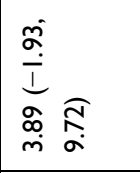 & 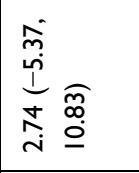 & 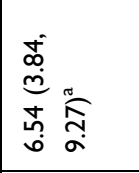 & 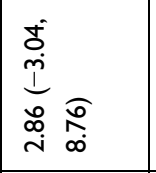 & 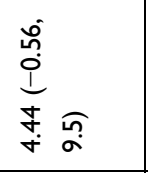 & $\begin{array}{l}\widehat{\Omega} \\
\underline{0} \\
\dot{0} \\
\bar{\infty}\end{array}$ \\
\hline 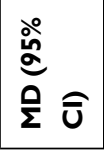 & $\sum$ & 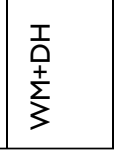 & $\sum \underset{\substack{x \\
⿱ 亠 乂}}{\stackrel{x}{N}}$ & $\begin{array}{l}\frac{\$}{3} \\
\sum_{3}^{+} \\
\sum\end{array}$ & $\sum \underset{+}{\vec{g}}$ & $\sum_{\sum}^{z} \underset{⿱ 亠 ⿻}{z}$ & $\begin{array}{l}\frac{u}{4} \\
\sum^{+} \\
3\end{array}$ & 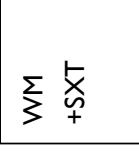 & 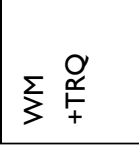 & $\sum_{3}^{\sum} \underset{x}{x}$ & $\begin{array}{l}\bar{P} \\
\text { D } \\
\sum_{j}^{+} \\
\end{array}$ & $\sum_{3} \stackrel{5}{x}$ \\
\hline
\end{tabular}




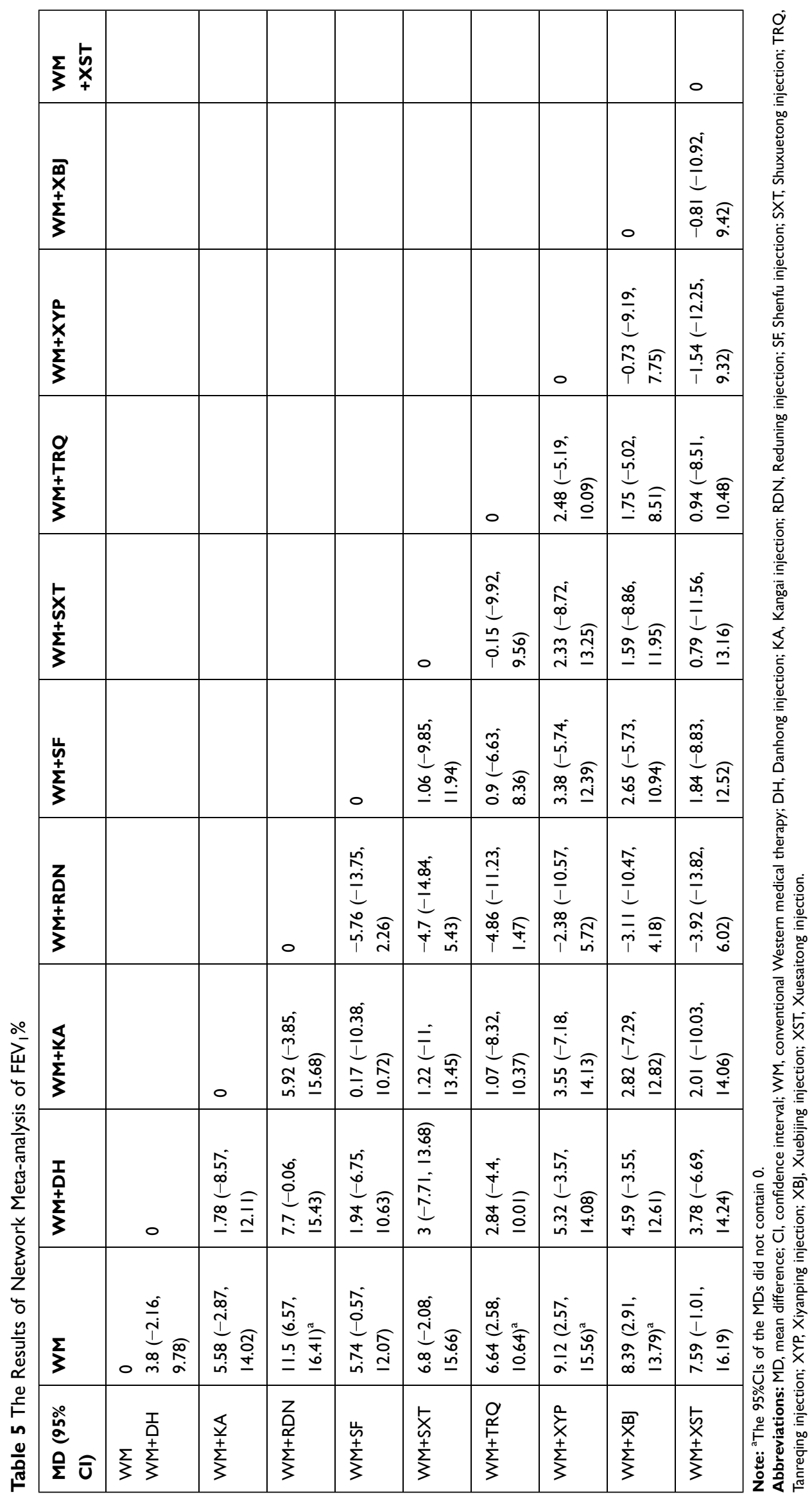


Table 6 The Results of Network Meta-analysis of Length of Hospital Stay

\begin{tabular}{|l|l|l|l|l|l|l|}
\hline MD (95\%Cl) & WM & WM+HQ & WM+SGM & WM+SM & WM+TRQ & WM+XYP \\
\hline WM & 0 & & & & & \\
WM+HQ & $-2.08(-5.83,1.67)$ & 0 & & & \\
WM+SGM & $-6.9(-10.89,-2.9)^{\mathrm{a}}$ & $-4.82(-10.28,0.68)$ & 0 & & \\
WM+SM & $-3.44(-7.42,0.55)$ & $-1.36(-6.82,4.11)$ & $3.46(-2.21,9.09)$ & 0 & \\
WM+TRQ & $-3.07(-4.97,-1.15)^{\mathrm{a}}$ & $-0.99(-5.18,3.24)$ & $3.83(-0.61,8.27)$ & $0.37(-4.03,4.79)$ & 0 \\
WM+XYP & $-3.4(-8.03,1.22)$ & $-1.32(-7.25,4.61)$ & $3.5(-2.61,9.59)$ & $0.04(-6.05,6.13)$ & $-0.33(-5.35,4.67)$ & 0 \\
\hline
\end{tabular}

Note: ${ }^{\text {a }}$ The $95 \% \mathrm{Cls}$ of the MDs did not contain 0 .

Abbreviations: MD, mean difference; $\mathrm{Cl}$, confidence interval; WM, conventional Western medical therapy; HQ, Huangqi injection; SGM, Shengmai injection; SM, Shenmai injection; TRQ, Tanreqing injection; XYP, Xiyanping injection.

reactions: nausea and vomiting ( $\mathrm{n}=16)$, fever $(\mathrm{n}=12)$, gastrointestinal reactions $(\mathrm{n}=6)$, rash $(\mathrm{n}=6)$, sweating $(\mathrm{n}=5)$, xerostomia $(\mathrm{n}=4)$, and chest distress $(\mathrm{n}=1)$. In contrast, of the 797 patients who received TCM injections plus WM, 55 patients $(6.90 \%)$ had the following adverse reactions: nausea and vomiting $(\mathrm{n}=11)$, fever $(\mathrm{n}=8)$, dizziness $(\mathrm{n}=4)$, xerostomia $(n=4)$, gastrointestinal reaction $(n=6)$, phlebitis $(n=4)$, chest distress $(n=1)$, epigastric discomfort $(n=2)$, bellyache $(\mathrm{n}=2)$, itchy skin $(\mathrm{n}=2)$, rash $(\mathrm{n}=2)$, allergy $(n=2)$, local pain during intravenous infusion $(n=2)$,
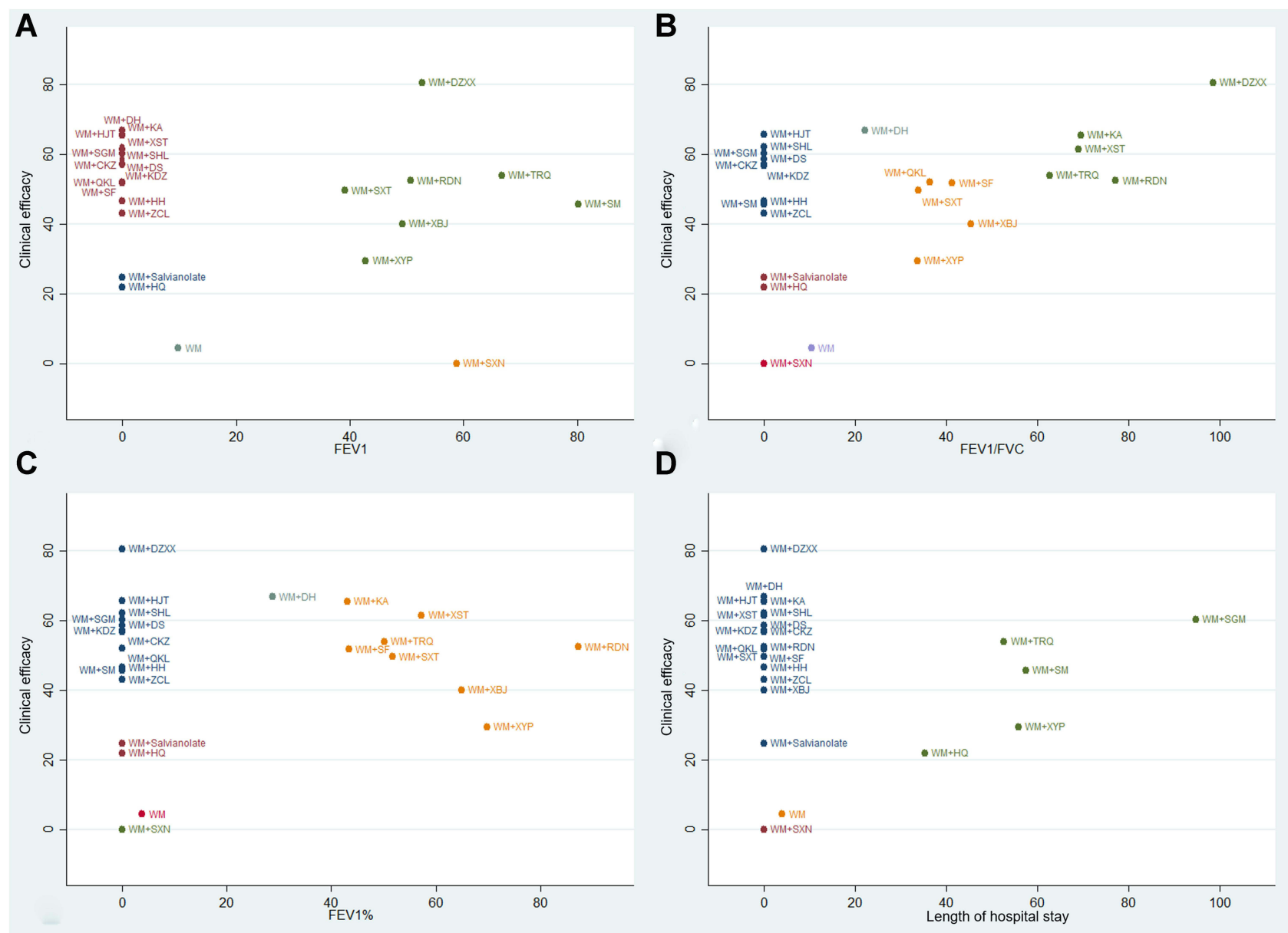

Figure 4 Cluster analysis plot for outcomes ((A) Clinical efficacy and FEV $($ (B) Clinical efficacy and FEV $/$ FVC (C) Clinical efficacy and FEV,\% (D) Clinical efficacy and length of hospital stay)).

Abbreviations: WM; conventional Western medical therapy; CKZ; Chuankezhi injection; DH; Danhong injection; DS; Danshen injection; DZXX; Dengzhanxixin injection; $\mathrm{HH}$; Honghua injection; HJT; Hongjingtian injection; HQ; Huangqi injection; KA; Kangai injection; KDZ; Kudiezi injection; QKL; Qingkailing injection; RDN; Reduning injection; SF; Shenfu injection; SGM; Shengmai injection; SM; Shenmai injection; SHL; Shuanghuanglian injection; SXN; Shuxuening injection; SXT; Shuxuetong injection; TRQ; Tanreqing injection; XYP; Xiyanping injection; XBJ; Xuebijing injection; XST; Xuesaitong injection; ZCL; Zhichuanling injection; Salvianolate; Salvianolate injection. 


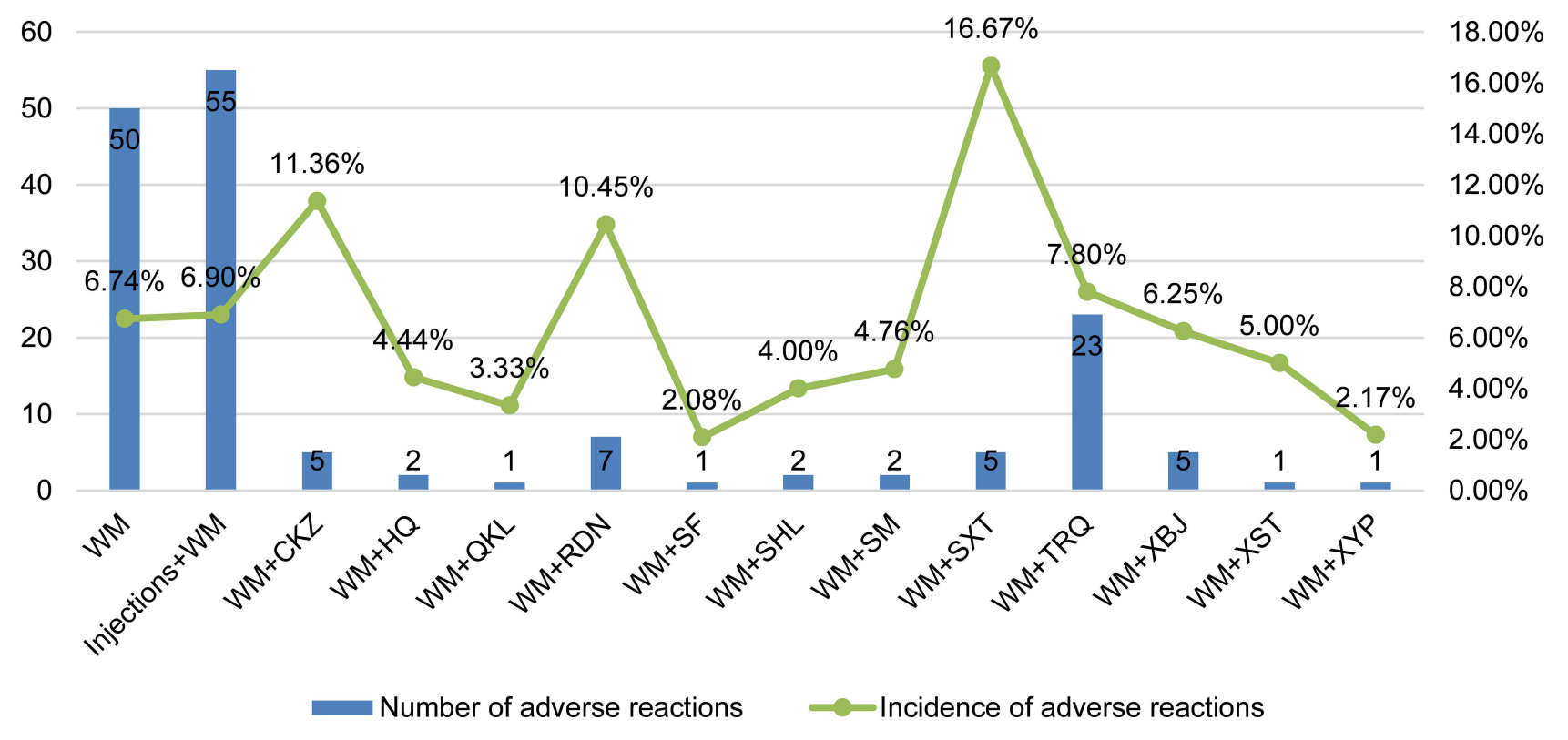

Figure 5 Number and incidence of adverse reactions of the included interventions.

Abbreviations: WM; conventional Western medical therapy; CKZ; Chuankezhi injection; HQ; Huangqi injection; QKL; Qingkailing injection; RDN; Reduning injection; SF; Shenfu injection; SM; Shenmai injection; SHL; Shuanghuanglian injection; SXT; Shuxuetong injection; TRQ; Tanreqing injection; XYP; Xiyanping injection; XBJ; Xuebijing injection; XST; Xuesaitong injection.

palpitation $(\mathrm{n}=1)$, headache $(\mathrm{n}=1)$, diarrhea $(\mathrm{n}=1)$, and dizziness + chest distress + xerostomia $(n=2)$. Of the 12 TCM injections, the highest incidence of adverse reactions was noted after WM+SXT (16.67\%), followed by WM $+\mathrm{CKZ}(11.36 \%)$ and $\mathrm{WM}+\mathrm{RDN}(10.45 \%)$. Adverse reactions were shown in Figure 5 and Supplementary Table S4. WM alone and TCM injections plus WM both had the following common adverse reactions: fever, nausea and vomiting, xerostomia, gastrointestinal reaction, and rash (Figure 6).

\section{Discussion}

This study included 103 RCTs, with 8767 patients and 23 CMIs including CKZ, DH, DS, DZXX, HH, HJT, HQ, KA, KDZ, QKL, RDN, SF, SGM, SM, SHL, SXN, SXT, TRQ, XYP, XBJ, XST, ZCL, and Salvianolate.

In patients with AECOPD, WM+DZXX had the highest likelihood of being the best treatment for improving both the clinical efficacy and $\mathrm{FEV}_{1} / \mathrm{FVC}, \mathrm{WM}+\mathrm{SM}, \mathrm{WM}$ $+\mathrm{RDN}$ and $\mathrm{WM}+\mathrm{SGM}$ had the highest likelihood of being the best treatment for improving $\mathrm{FEV}_{1}, \mathrm{FEV}_{1} \%$, and length of hospital stay, respectively.

The cluster analysis revealed that WM+DZXX had the most favorable response for clinical efficacy and $\mathrm{FEV}_{1}$, as well as clinical efficacy and $\mathrm{FEV}_{1} / \mathrm{FVC}, \mathrm{WM}+\mathrm{RDN}$ had the most favorable response for clinical efficacy and
$\mathrm{FEV}_{1} \%$, WM+SGM had the most favorable response for clinical efficacy and length of hospital stay.

DZXX is a sterile aqueous solution composed of Erigerontis Herba extract, has been used in China for many years. Its main active components include flavonoids and phenolic acids. ${ }^{31}$ Flavonoids can activate blood and dissolve stasis as well as inhibit the inflammatory reaction in the lung and the synthesis of collagen fiber to prevent pulmonary fibrosis. ${ }^{32}$ Clinical studies have shown that compared with WM alone, DZXX achieved better efficacy when administered to patients with moderately severe COPD, it could not only reduce inflammation, but also improve hemorheological indicators and lung function. ${ }^{33}$ Experimental studies show that DZXX can decrease transforming growth factor $\beta 1$ activity to inhibit fibroblast proliferation, collagen fiber and extracellular matrix synthesis, delaying or improving the process of airway remodeling and irreversible obstruction in COPD. ${ }^{34-36}$

RDN is composed of Artemisiae Annuae Herba, Lonicerae Japonicae Flos, and Gardeniae Fructus, is generally administered as an intravenous injection to treat cold, cough, upper respiratory infections, and acute bronchitis, and it has a good curative effect in clinics. ${ }^{37,38}$ Previous studies have shown that cryptochlorogenic acid, neochlorogenic acid, and geniposide - the main active substances of $\mathrm{RDN}^{39-44}$ — can increase superoxide dismutase (SOD) activity, suppress myeloperoxidase (MPO) 


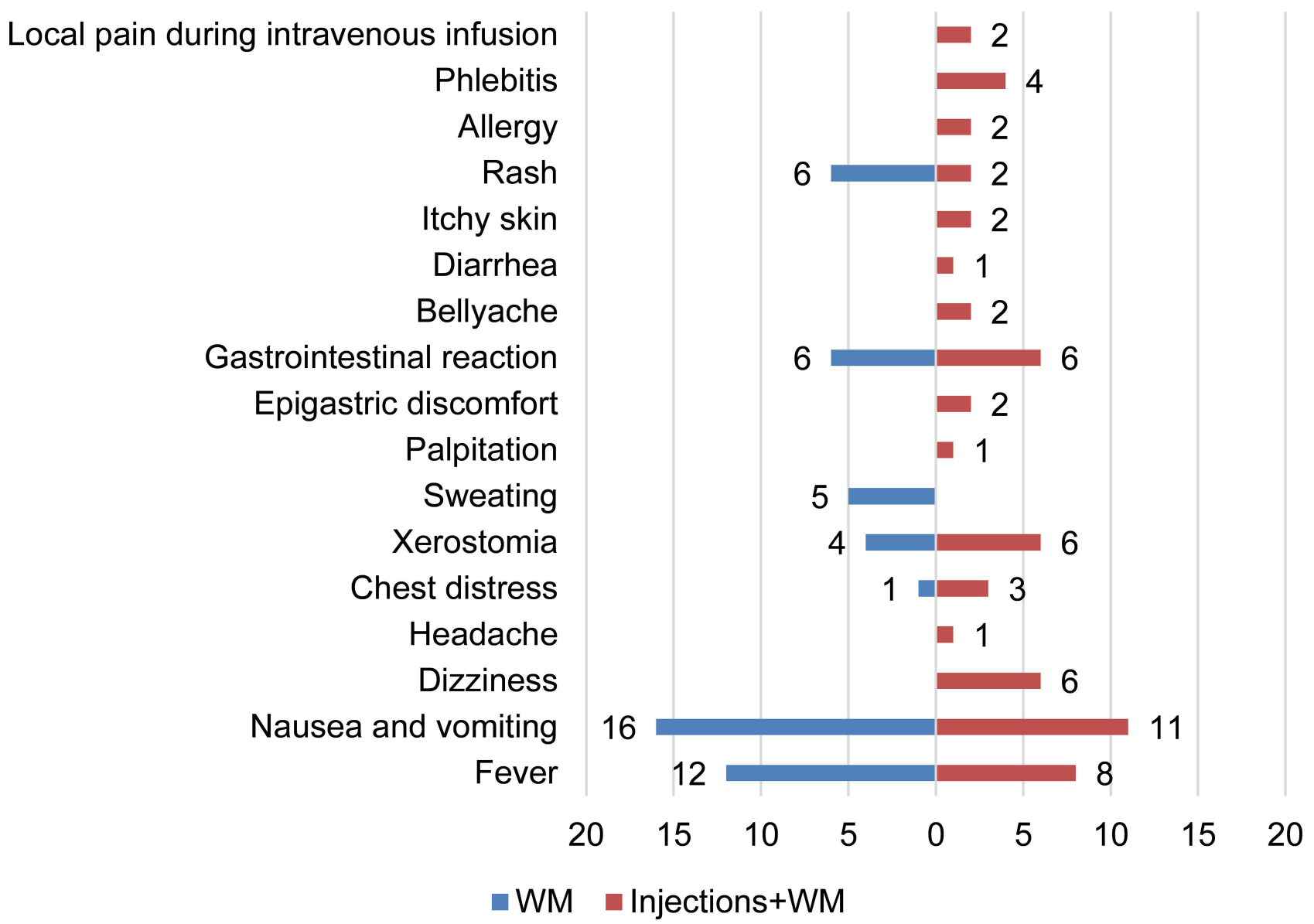

Figure 6 Adverse reactions after WM alone and TCM injections plus WM. Abbreviation: WM; conventional Western medical therapy.

activity, and reduce the wet/dry (W/D) ratio and total leukocyte and neutrophil numbers, ${ }^{38}$ it can thus be antiinflammatory, improve immunity, and alleviate damage caused by the diseases. ${ }^{45,46}$ In addition, a network analysis identified two key compounds (CFA and ferulic acid), five key targets (Bcl-2, eNOS, PTGS2, PPARA, and MMPs), and four key pathways (estrogen signaling pathway, PI3KAKT signaling pathway, cGMP-PKG signaling pathway, and calcium signaling pathway) for RDN-all of which play critical roles in the treatment of inflammatory diseases. ${ }^{47}$

The normal respiratory movement of the human body involves the joint participation of nerve cells that produces the respiratory rhythm and those that regulate the respiratory movement in the central nervous system. ${ }^{48}$ Studies have shown that the irreversible airflow limitation of COPD may be related to the abnormal excitability of the respiratory center. ${ }^{48}$ SGM is composed of Red Ginseng, Ophiopogonis Radix, and Schisandrae Chinensis
Fructus. Here, Ophiopogonis Radix nourishes the yin (nutrition and fluid in the human body, which nourishes various organs $^{49}$ ), Schisandrae Chinensis Fructus astringents the qi (vital energy, regarded as a driving force of biological activities in the human body, including both nutrient substances and organ functions ${ }^{50}$ ) and has antitussive effects, and Red Ginseng tonifies qi and enhances immunity. ${ }^{51}$ The combination of these herbs affects the respiratory center and then relieves dyspnea in COPD patients. ${ }^{52,53}$ Modern studies have also indicated that SGM can improve pulmonary ventilation function, thus increasing the alveolar diffuse area, adjusting the airflow ratio, reducing myocardial oxygen consumption and glucose metabolism, and enhancing gland and endocrine function, as a result, the whole body function is adjusted, qi becomes tonified and blood is activated. ${ }^{54} \mathrm{~A}$ meta-analysis reported that $\mathrm{SGM}+\mathrm{WM}$ has significant efficacy in COPD treatment, where it improves clinical efficacy and lung function, regulates immune function, and shortens disappearance time of lung rales. ${ }^{55,56}$ 
Adverse reactions appeared in both treatment group and control group of included studies. However, the specific correlation between the TCM injections used and adverse reactions could not be determined. The incidence of adverse reactions was high in WM+SXT (16.67\%), $\mathrm{WM}+\mathrm{CKZ}(11.36 \%)$, and $\mathrm{WM}+\mathrm{RDN}(10.45 \%)$, compared with WM alone. Thus, the safety of CMIs still needs further evaluation.

\section{Limitations}

The number of original studies on this research topic met the basic requirements for this NMA, but the quality of these studies were not high. In particular, the limitations of our study were as follows:

(1) Only $31.07 \%$ of the studies used the correct random method, which may have resulted in selective biases.

(2) Most of the studies did not mention the blinding of participants or personnel and allocation concealment, which may have resulted in implementation biases.

(3) Of all the included studies, $15.53 \%$ merely used subjective indicators as the outcome evaluation index, which may have resulted in measurement bias.

(4) The 103 included studies did not mention protocol registration and conflict of interests, therefore, the sources of other bias could not be determined.

(5) The funnel plot for clinical efficacy indicated the possibility of publication bias. The missing contents from ongoing studies and gray literature may result in publication bias. ${ }^{55}$

(6) None of the included studies restricted the TCM syndromes of AECOPD patients. However, patients with different TCM syndromes who were treated with the same intervention may not represent the real effect of the TCM drugs.

(7) The participant age and treatment duration varied in the included studies, which may have affected the stability of results.

(8) All included studies were conducted in China, this might weaken the generalization of the results.

\section{Conclusion}

In conclusion, WM+DZXX had the highest likelihood of being the best treatment for improving both the clinical efficacy and $\mathrm{FEV}_{1} / \mathrm{FVC}, \mathrm{WM}+\mathrm{SM}, \mathrm{WM}+\mathrm{RDN}$ and $\mathrm{WM}$ +SGM had the highest likelihood of being the best treatment for improving $\mathrm{FEV}_{1}, \mathrm{FEV}_{1} \%$ and length of hospital stay, respectively. Combined with cluster analysis results, DZXX, RDN or SGM plus WM were noted to be the optimum treatment regimens for improving the condition of patients with AECOPD. However, the quality of studies evaluating the efficacy of various CMIs is not good. Therefore, additional high-quality studies are warranted.

\section{Abbreviations}

COPD, chronic obstructive pulmonary disease; AECOPD, acute exacerbation of chronic obstructive pulmonary disease; TCM, traditional Chinese medicine; CMI, Chinese medical injection; RCT, randomized controlled trial; CKZ, Chuankezhi injection; DH, Danhong injection; DS, Danshen injection; DZXX, Dengzhanxixin injection; HH, Honghua injection; HJT, Hongjingtian injection; HQ, Huangqi injection; KA, Kangai injection; KDZ, Kudiezi injection; QKL, Qingkailing injection; RDN, Reduning injection; SF, Shenfu injection; SGM, Shengmai injection; SM, Shenmai injection; SHL, Shuanghuanglian injection; SXN, Shuxuening injection; SXT, Shuxuetong injection; TRQ, Tanreqing injection; XYP, Xiyanping injection; XBJ, Xuebijing injection; XST, Xuesaitong injection; ZCL, Zhichuanling injection; Salvianolate, Salvianolate injection; WM, conventional Western medical therapy; ADR, adverse reaction; $\mathrm{FEV}_{1}$, forced expiratory volume in one second; $\mathrm{FEV}_{1} / \mathrm{FVC}$, ratio of forced expiratory volume in one second to forced vital capacity; $\mathrm{FEV}_{1} \%$, ratio of forced expiratory volume in one second to the predicted value.

\section{Data Sharing Statement}

The raw data supporting the conclusion of this article will be made available by the corresponding author (Hui Wang) without undue reservation.

\section{Ethics Approval and Consent to Participate}

This study is an overview of the literature thus ethics approval was not needed.

\section{Consent to Publish}

The study group consented to publish.

\section{Acknowledgments}

Thanks to the authors of the included studies to provide primary data.

\section{Funding}

This overview was funded by the Special Support Plan for Talent Development of Tianjin-Young Top Talent Project (No. 201504)/ Training Program of Innovation Team of 
Tianjin Higher Education Institution through Tianjin Municipal Education Commission (No. TD13-5047).

\section{Disclosure}

The authors report no conflicts of interest in this work.

\section{References}

1. Rodriguez-Roisin R. Toward a consensus definition for COPD exacerbations. Chest. 2000;117(5):398S-401S. doi:10.1378/ chest.117.5_suppl_2.398S

2. Burge S, Wedzicha JA. COPD exacerbations: definitions and classifications. Eur Respir J Suppl. 2003;21(Supplement41):46s53s. doi:10.1183/09031936.03.00078002

3. Pavord ID, Jones PW, Burgel PR, Rabe KF. Exacerbations of COPD. Int J Chron Obstruct Pulmon Dis. 2016;11:21-30. doi:10.2147/COPD.S85978

4. Celli BR, Thomas NE, Anderson JA, et al. Effect of pharmacotherapy on rate of decline of lung function in chronic obstructive pulmonary disease: results from the TORCH study. $A m ~ J$ Resp Crit Care. 2008;178(4):332-338. doi:10.1164/rccm.200712$18690 \mathrm{C}$

5. Min L, Yang Z, Dan-Dan L, Jing S. Tranditional care interventions to reduce readmission in patients with chronic obstructive pulmonary disease: a meta-analysis of randomized controlled trials. Chin Nursing Res. 2017;4(2):84-91. doi:10.1016/j. cnre.2017.06.004

6. Milavetz G. Global Surveillance; Prevention and Control of Chronic Respiratory Diseases: a Comprehensive Approach. $J$ Pharm Tech. 2008;24(2):122.

7. Wedzicha JA, Seemungal TAR. COPD exacerbations: defining their cause and prevention. Lancet. 2007;370(9589):786-796. doi:10.1016/S0140-6736(07)61382-8

8. Martinez FJ, Han MK, Flaherty K, Curtis J. Role of infection and antimicrobial therapy in acute exacerbations of chronic obstructive pulmonary disease. Expert Rev Anti Infect Ther. 2006;4 (1):101-124. doi:10.1586/14787210.4.1.101

9. Lim S, Lam DC, Muttalif AR, et al. Impact of chronic obstructive pulmonary disease (COPD) in the Asia-Pacific region: the EPIC Asia population-based survey. Asia Pac Fam Med. 2015;14(1):4. doi:10.1186/s12930-015-0020-9

10. Shilian H, Jing W, Cui C, Xinchun W. Epidemiological analysis of chronic diseases in Chinese residents. Chin J Clin Healthcare. 2020;23(3):8-13.

11. Writing Group of Expert Consensuson on Anti-infective Therapy for Acute Exacerbation of Chronic Obstructive Pulmonary Disease. Expert consensusonanti-infective therapy for acute exacerbation of chronic obstructive pulmonary disease in China. Int J Respir. 2019;39(17):1281-1296.

12. Chen W, Jianying X, Lan Y, et al. Prevalence and risk factors of chronic obstructive pulmonary disease in China (the China Pulmonary Health $[\mathrm{CPH}]$ study): a national cross-sectional study. Lancet. 2018;391(10131):1706-1717. doi:10.1016/S01406736(18)30841-9

13. Yao C, Liu X, Tang Z. Prognostic role of neutrophil-lymphocyte ratio and platelet-lymphocyte ratio for hospital mortality in patients with AECOPD. Int J Chronic Obstr. 2017;12:2285-2290.

14. Global Initiative For Chronic Obstructive Lung Disease. Global strategy for the diagnosis; management; and prevention of chronic obstructive pulmonary disease (2021report). Available from: https://goldcopd.org/2021-gold-reports/. Accessed August $18,2021$.
15. Polverino F, Kheradmand F. COVID-19; COPD; and AECOPD: immunological; Epidemiological; and Clinical Aspects. Front Med. 2021;7:627278. doi:10.3389/fmed.2020.627278

16. Wang L, Fan Y, Xu J, Deng H, Jia B. The efficacy and safety of Tanreqing injection combined with western medicine for severe pneumonia: a protocol for systematic review and meta-analysis. Medicine. 2020;99(35):e22010. doi:10.1097/MD.0000000000022 010

17. Xianghua G. The research progress of COPD with catabasis. Nei Mongol J Traditional Chin Med. 2017;18(36):123-124.

18. Peng YQ, Mao YM, Zhu JQ, et al. A clinical study of short-term Xuebijing injection on treatment of patients with acute exacerbation of chronic obstructive pulmonary disease. Chin J Integr Traditional Western Med Intensive Critical Care. 2008;3:178180.

19. Duan L, Ning C. Observation Curative Effect of Xuebijing Injection Synergistic in Treatment of Acute Exacerbation of Chronic Obstructive Pulmonary Disease. China Continuing Med Educ. 2015;7(31):219-220.

20. Xie S, Yan P, Yao C, et al. Efficacy and safety of Xuebijing injection and its influence on immunomodulation in acute exacerbations of chronic obstructive pulmonary disease: study protocol for a randomized controlled trial. Trials. 2019;20(1):136. doi:10. 1186/s13063-019-3204-z

21. Chen SQ, Yang XY, Tang XY, et al. Systematic review of Chuankezhi injection for treating acute exacerbation of chronic obstructive pulmonary disease. China J Chin Materia Medica. 2017;42(14):2789-2795. doi:10.19540/j.cnki.cjcmm.20170523. 009

22. Gao LN, Lyu J, Wang ZF, Yu DD, Sun MH. Meta-analysis of randomized controlled trials on effect of Tanreqing Injection combined with Western medicine on acute exacerbation of chronic bronchitis. China j Chin materia medica. 2019;44 (24):5313-5321. doi:10.19540/j.cnki.cjcmm.20190924.501

23. Huang X, Duan X, Wang K, Wu J, Zhang X. Shengmai injection as an adjunctive therapy for the treatment of chronic obstructive pulmonary disease: a systematic review and meta-analysis. Complement Ther Med. 2019;43:140-147. doi:10.1016/j. ctim.2019.01.020

24. Gong G, Li X. Study of clinical effect on treatment of chronic obstructive pulmonary disease in acute aggravated stage with Tanreqing injection and cell factor level. China $j$ Chin materia medica. 2009;34(1):104-106.

25. Wang TJ, Xie ZH, Zhao ZZ. Effects of shuxuening injection on the levels of serum matrix metalloproteinase- 9 and tissue inhibitor of metalloproteinase-1 in acute exacerbated chronic obstructive pulmonary disease patients. Chin $j$ Integrated Trad Western Med. 2012;32(2):191-194.

26. Chinese Sociey of Respiratory Diseases. Diagnosis and treatment guidance of chronic obstructive pulmonary disease. Chin $J$ Tuberculosis Respir Dis. 2013;36(4):255-264.

27. Hosseini HM, Pai DR, Ofak DR. COPD: does Inpatient Education Impact Hospital Costs and Length of Stay? Hosp Top. 2019;97(4):165-175. doi:10.1080/00185868.2019.1677540

28. Wedzicha JA, Brill SE, Allinson JP, Donaldson GC. Mechanisms and impact of the frequent exacerbator phenotype in chronic obstructive pulmonary disease. BMC Med. 2013;11(1):181. doi:10.1186/1741-7015-11-181

29. Higgins JP, Altman DG, Gøtzsche PC, et al. The Cochrane Collaboration's tool for assessing risk of bias in randomised trials. BMJ. 2011;343(oct182):d5928. doi:10.1136/bmj.d5928

30. Hutton B, Salanti G, Caldwell DM, et al. The PRISMA extension statement for reporting of systematic reviews incorporating network meta-analyses of health care interventions: checklist and explanations. Ann Intern Med. 2015;162(11):777-784. doi:10. 7326/M14-2385 
31. Hua F, Peng L, Qiang K, Zhi-Long Z, Ji W, Jia-Yi C. Metabolism and Pharmacological Mechanisms of Active Ingredients in Erigeron breviscapus. Curr Drug Metab. 2021;22(1):24-39. doi:10.2174/1389200221666201217093255

32. Juan J, Qiangjin G, Nianzhi Z. Experimental Research Progress in Chinese Herbal Medicines with the Effects of Benefiting Qi and Activating Blood Circulation and Their Active Ingredients in Treating Pulmonary Fibrosis. Chin J Inf TCM. 2015;22(9):131134.

33. Junping S. Hemorheology and pulmonary function analysis of Erigeron breviscapus injection in the treatment of moderate and severe chronic obstructive pulmonary disease in the elderly during stable period. J Electrocardiogram. 2018;7(4):101-103.

34. Fei D, Gang H, Daigang C, Huajun Y. Effects of Breviscapine on airway remodeling in rats with chronic obstructive pulmonary disease model. Hebei J Trad Chin Med. 2017;39(7):1069-1073.

35. Chua F, Sly PD, Laurent GJ. Pediatric lung disease: from proteinases to pulmonary fibrosis. Pediatr Pulm. 2005;39(5):392-401. doi:10.1002/ppul.20171

36. Willis BC, Borok Z. TGF-beta-induced EMT: mechanisms and implications for fibrotic lung disease. Am J Physiol Lung Cell Mol Physiol. 2007;293(3):L525-34. doi:10.1152/ajplung.00163.2007

37. Guoliang Z, Jie Z, Liyun H, Shiyan Y, Ziqiang Z. Reduning injection for fever; rash; and ulcers in children with mild hand; foot; and mouth disease: a randomized controlled clinical studyScienceDirect. J Tradit Chin Med. 2013;33(6):733-742. doi:10. 1016/S0254-6272(14)60005-4

38. Luping T, Wei X, Yifang L, et al. Anti-inflammatory effects of Reduning Injection on lipopolysaccharide-induced acute lung injury of rats. Chin J Integr Med. 2014;20(8):591-599. doi:10.1007/s11655-014-1758-x

39. Gao W, Wang S, Cui Z, Cao J, Tian H. Reduning injection for community-acquired pneumonia: meta-analysis. China $J$ Chin Materia Medica. 2011;36(24):3539.

40. Chang XJ, Zhang S, Chen J, et al. Effect of Reduning Injection on Acute Lung Injury of Rats. Chin J Exp Trad Med Formulae. 2014;20(8):591-599.

41. Jian C, Xiujuan C, Chunmiao C, et al. Study on Analgesic and Antipyretics Effect of Re-Du-Ning Injection. Modernization Traditional Chin Med Materia Medica World Sci Tech. 2014;16 (9):1912-1915.

42. Sha W, Ye J, Qian L, Liu QA, Wei X. On-line quantitative monitoring of liquid-liquid extraction of Lonicera japonica and Artemisia annua using near-infrared spectroscopy and chemometrics. Pharmacogn Mag. 2015;11(43):643-650. doi:10.4103/ 0973-1296.160465

43. Fang W, Li CY, Zheng YF, Li HY, Xiao W, Peng GP. Identification of the Allergenic Ingredients in Reduning Injection by Ultrafiltration and High-Performance Liquid Chromatography. J Immunol Res. 2016;2016(2):1-7.

44. Wu RS, Tao L, Qin PP, Ling Y, Yan Z. Physical fingerprint for quality control of Reduning injection. China j Chin materia medica. 2017;42(3):505. doi:10.19540/j.cnki.cjcmm.20170103.020

45. Yejing Z. Effect of Reduning Injection Combined with Cefotaxime Sodium on Chronic Obstructive Pulmonary Disease Complicated with Pulmonary Infection. J Qiqihar Med Univ. 2018;39(24):2899-2901.

46. Yufeng W, Zhongbin Z. Therapeutic effect of Reduning injection on acute upper respiratory tract infection. Evaluation Analysis Drug Use Hospitals China. 2017;17(8):1052-1053.

47. Fuda X, Mingxiang X, Yibing Y, et al. Assessing the Anti-inflammatory Mechanism of Reduning Injection by Network Pharmacology. Biomed Res Int. 2020;2020(4):1-13.

48. Jolley CJ, Luo YM, Steier J, Reilly C, Moxham J. Neural respiratory drive in healthy subjects and in COPD. Eur Respir J. 2009;33(2):289-297. doi:10.1183/09031936.00093408
49. Kang YM, Seo MG, Lee KY, An HJ. Antiphotoaging Potential of Extracts of Yin-Tonic Herbal Medicine in Skin Cell and Human Skin Equivalent. Evid Based Compl Alt. 2020;20(2020):8881270.

50. Wang XM, Xiao-Bo LI, Peng Y. Impact of Qi -invigorating traditional Chinese medicines on intestinal flora: a basis for rational choice of prebiotics. Chin J Nat Med. 2017;15(4):241254. doi:10.1016/S1875-5364(17)30041-9

51. Qing F. Effects of Shenmai injection on serum migration inhibitory factor and tuillor necrosis factor- $\mathrm{d}$ levels in patients with acute viral myocarditis and its significance. Chin $j$ Ethnomed Ethnopharm. 2010;19(5):31-32.

52. Xiaoli L, Xiaoyun L, Zhongtao L, et al. Effect of Shengmai Injection on Phrenic Nerve Discharge of COPD rats. $J$ Shaanxi Univ Chin Med. 2017;40(2):71-74.

53. Haopeng $\mathrm{Y}$, Long L, Chengzhi C, Junping K, Boyang Y, Yongqing Y. Neuroprotective Effect of Shengmai Injection on Expression of Tissue Factor and related Signal Pathways in Mice with Cerebral Ischemia-reperfusion Injury. Chin $J$ Exp Trad Med Formulae. 2013;19(14):194-199.

54. Guangrong N. Shengmai Injection Combined with Western Medicine in the Treatment of Lung and Kidney Yin Deficiency of Two Chronic Obstructive Pulmonary Disease Randomized Controlled Study. $J$ Pract Traditional Chin Internal Med. 2016;30(12):69-71.

55. Xingyue H, Xiaojiao D, Kaihuan W, Jiarui W, Xiaomeng Z. Shengmai injection as an adjunctive therapy for the treatment of chronic obstructive pulmonary disease: a systematic review and meta-analysis. Complement Ther Med. 2019;43(1):140-147.

56. Chen L, YingJia LU. Influence of Shengmai Injection on the Inflammatory Factors of Patients with Chronic Obstructive Pulmonary Disease. Henan Traditional Chin Med. 2016;36(3):538539.

57. Lili C, Jinhui L, Demei L, Jiangang X. Clinical observation of Kang'ai injection in the treatment of elderly patients with AECOPD. J Clin Pulmonary Med. 2014;19(7):1247-1250.

58. Weizhong C, Ji L. Efficacy of Reduning Injection on AECOPD:A Clinical Observation of 77cases. Guiding J Traditional Chin Med Pharm. 2014;20(13):69-71.

59. Zhu C, Zhong L. Influence of Danghong injection on TNF-alpha and IL- 8 of patients with chronic obstructive pulmonary disease in acute exacerbation. J Liaoning Med Univ. 2013;34(1):23-25.

60. Yongsheng C. Clinical observation of Shenfu injection in adjuvant treatment of acute exacerbation of chronic obstructive pulmonary disease. J Em Trad Chin Med. 2015;24(3):553-554.

61. Yandong C, Junhe W. The ameliorative effect of Chuankezhi injection on acute exacerbation of chronic obstructive pulmonary disease. Clin J Traditional Chin Med. 2010;22(5):419-420.

62. Hongzhen D, Wei Z. The Clinical Curative Effect of Reduning Injection treating Acute Exacerbation of Chronic Obstructive Pulmonary Disease (Syndrome of Phlegm Heat Obstructing in the Lung). J Em Trad Chin Med. 2015;24(8):1433-1435.

63. Du J. Effect of Danhong injection on acute exacerbation of chronic obstructive pulmonary disease. China Practical Med. 2014;9(32):135-136.

64. Yong D, Zhijun J, Wei H. Study of influence of Tanreqing injection on serum high-sensitivity C- reactive protein and procalcitonin of patients with acute exacerbation of chronic obstructive pulmonary disease. Chin J Nosocomiol. 2015;25(6):1233-1235.

65. Limin D, Chaozhi N. Observation Curative Effect of Xuebijing Injection Synergistic in Treatment of Acute Exacerbation of Chronic Obstructive Pulmonary Disease. China Continuing Med Educ. 2015;7(31):219-220.

66. Yongxia F. Observation of curative effect of Danhong injection on acute attack of COPD. $J$ North Pharm. 2011;8(9):17-18.

67. Qing F. Observation on the curative effect of Tanreqing on acute exacerbation of chronic obstructive pulmonary disease. $J$ Clin Ration Drug Use. 2015;8(7B):48-49. 
68. Zhijun F, Wei T. Effects of Tanreqing Injection on Expression of Serum C-reactive Protein (CRP); IL-8 and IL-17 in Patients with Acute Exacerbation of COPD. China Foreign Med Treat. 2010;29 (19):83-84.

69. Dongwei F. Clinical observation of Tanreqing injection in the treatment of acute exacerbation of chronic obstructive pulmonary. J Em Trad Chin Med. 2012;21(8):1298.

70. Qin F, Li L, Fang T. Tanreqing combined with antibiotics in the treatment of acute exacerbation of chronic obstructive pulmonary disease in elderly patients. J Em Trad Chin Med. 2009;18(1):120-121.

71. Yongwang F. Clinical observation of Tanreqing injection in the treatment of acute exacerbation of COPD. China Med Herald. 2009;6(33):147.

72. Zongkai G. Analysis of curative effect of Tanreqing injection on acute exacerbation of chronic obstructive pulmonary disease. China Practical Med. 2015;10(33):194-195.

73. Zongkai G. Application value of Danhong injection in the treatment of acute episode of COPD. Med Forum. 2016;20(9):1190-1191.

74. Xia G, Daiqiu P. Clinical effect of integrated traditional Chinese and western medicine on acute exacerbation of chronic obstructive pulmonary disease. Chin J Modern Drug App. 2009;3 (15): $153-154$

75. Fang H, Xin Z. Efficacy of Xiyanping in the treatment of acute exacerbation of chronic obstructive pulmonary disease. Mod $J$ Integr Trad Chin Western Med. 2015;24(8):870-872.

76. Tianpao H, Feiyu L, Yinru L. Clinical Observation of Xuebijing treating Acute Exacerbation of Chronic Obstructive Pulmonary Diseases. Chin Arch Traditional Chin Med. 2010;28(10):2232-2233.

77. Shaoling H. Efficacy of Xuebijing injection in treating acute exacerbation period of chronic obstructive pulmonary disease patients and the effect on levels of SP-D and CCL18. Jilin Med J. 2019;40(5):925-927.

78. Xiang H. Clinical Observation on Tanreqing Injection combined with Conventional Western Medicine in Treatment of Chronic Obstructive Pulmonary Disease in Acute Exacerbation. $J$ Hubei Univ Chin Med. 2016;18(5):74-76.

79. Yongliang H, Juhua M. Observation of effect of Danshen injection on acute exacerbation of chronic obstructive pulmonary disease. J Clin Ration Drug Use. 2012;5(19):100-101.

80. Xiaolin H, Xiaobin D, Zhenghua K, Jing T, Shiying Z. Comparison of Tanreqing and Xuebijing in treatment of acute exacerbation of chronic obstructive pulmonary disease. Drugs Clinic. 2016;31(11):1732-1736.

81. Wenshan H. Tanreqing injection in treating 20 cases of acute onset of chronic obstructive pulmonary disease. Hunan $J$ Traditional Chin Med. 2014;30(2):32-34.

82. Bin H, Qixiang H, Dongsheng L, Shanmao X. Clinical study of Tanreqing injection in the treatment of acute exacerbation of chronic obstructive pulmonary. J Em Trad Chin Med. 2006;15 (5):464-466.

83. Jin K, Yi Y. The effect analysis of phlegm heat injection auxiliary treatment of elderly AECOPD. J Front Med. 2016;6(26):30-31.

84. Daixiang L. Clinical efficacy of Xuebijing in the treatment of acute exacerbation of chronic obstructive pulmonary disease (AECOPD). Capital Med. 2015;22(8):47.

85. Guolin L, Nianzhi Z, Wei R. Clinical observation of Tanreqing in the treatment of AECOPD. J Clin Pulmonary Med. 2009;14(3):416.

86. Linlin L, Qianlong X, Baoli X, Yunfeng L, Shuchi H. Clinical observation of Tanreqing in treating patients with acute onset of chronic obstructive pulmonary disease. J Ningxia Med Coll. 2014;36(2):204-206.

87. Wen LI, Bing M, Gang W, et al. Effect of Tanreqing injection on treatment of acute exacerbation of chronic obstructive pulmonary disease with Chinese medicine syndrome of retention of phlegm and heat in Fei. Chin J Integr Med. 2010;16(2):131-137. doi:10.1007/s11655-010-0131-y
88. Gang L. Cfinical observation of Danshen injection in adjuvant treating patients with acute exacerbations of chronic obstructive pulmonary disease. Nei Mongol J Traditional Chin Med. 2009;28 (14):21-22.

89. Wei L, Sining C, Ruixiang L. Effects of Tanreqing Injection on hs-CRP, IL-6, IL-10 in acute exacerbation of Chronic Obstructive Pulmonary Disease. J Guangxi Univ Chin Med. 2017;20(1):1113.

90. Wensheng L, Weiqing L, Shiwei C, Qingshun H. Influence of Shenfu injection on tumor necrosis factor-a, interleukin-2 and lung function In patients with chronic obstructive pulmonary disease at acute exacerbation stage. Chin J Integr Traditional Western Med Intensive Critical Care. 2008;15(3):149-151.

91. Daobo L, Hongli Y, Wenteng C, Min L. Effect of Xuebijing injection on chronic obstructive pulmonary disease and blood coagulation function. China Trop Med. 2009;9(8):1514-1515.

92. Hongbo L. Clinical observation of Tanreqing in treating 60 cases of acute onset of chronic obstructive pulmonary disease. $J \mathrm{Em}$ Trad Chin Med. 2008;17(4):448-449.

93. Honghong L, Shirong D, Jin L, Aiinn L. The clinical observations of Xiyanping as an adjuvant therapy on acute exacerbation of chronic obstructive pulmonary disease. J Trop Med. 2016;16 (10):1293-1295.

94. Yan L, Lili D, Binbin Q, et al. Effect of Shuxuetong Injection on "Inflammatory Factor - Vascular Endothelial Function Hemorheology" Network in AECOPD Patients. Mod J Integr Trad Chin Western Med. 2019;28(2):165-169.

95. Zhanxiang L, Anmeng W. Effect of Tanreqing injection in treatment of 100 patients with the exacerbation of chronic obstructive pulmonary disease. China Pract J Med. 2007;34(24):27-28.

96. Zonggui L. Analysis of curative effect of Tanreqing injection on acute exacerbation of chronic obstructive pulmonary disease. Henan Med Res. 2014;23(3):121-123.

97. Hai L. Clinical observation on tanreqing injection in treating 124 cases of acute exacerbation of chronic obstructive pulmonary. $J$ Em Trad Chin Med. 2012;21(6):966.

98. Na L, Meixia W, Lina D, Shuyuan A. Clinical Observation of Tanreqing Injection in Treatment for Acute Exacerbation of Chronic Obstructive Pulmonary Disease in the Elderly Respiratory Ward. Guide China Med. 2013;11(24):420-421.

99. Lin M. Observation of curative effect of Xuebijing injection on acute exacerbation of chronic obstructive pulmonary disease. Chin J Clin Rational Drug Use. 2014;7(9):142-143.

100. Lijian P, Zang N, Liu C, Zheng W, Lu X. Anti-inflammatory and Immunization Properties of Re-Du-Ning Injection in Treatment of AECOPD with Phlegm-heat Stagnated in the Lung Syndrome. Modernization Traditional Chin Med Materia Medica World Sci Tech. 2015;17(6):1225-1229.

101. Bo P, Nianzhi Z, Hongyan J, Guolin L, Wei R, Wei C. Effect of Tanreqing on pulmonary function of acute exacerbation of COPD (Syndrome of retention of phlegm and heat in Fei). J Em Trad Chin Med. 2007;16(12):1456-1457.

102. Qin Q, Xinjun F, Yang P, et al. Clinical research of Xuebijing parenteral solution on the coagulation status and lung function of patients with acute exacerbation of chronic obstructive pulmonary disease. Chin J Critical Care MeD. 2013;33(6):543-545.

103. Qiu Q, Jian X, Shuyun L. Therapeutic Effect of Sofren Injection in Treatment of Acute Exacerbations of COPD. Chin Med Innovations. 2014;11(32):14-16.

104. Yuejuan R, Xuemei L, CMmei Z. Clinical observation of Shenfu Injection in the treatment of acute exacerbation chronic obstructive pulmonary disease. Nei Mongol J Traditional Chin Med. 2013;32(7):58-59.

105. Ce S. Tanreqing injection in the treatment of 50 cases of acute chronic obstructive pulmonary disease. China Pract J Med. 2009;36(9):54-55. 
106. Daihui S. Evaluation on the efficacy of Tanreqing on 42 patients with chronic obstructive pulmonary disease of acute exacerbation. Youjiang Med J. 2013;41(1):23-24.

107. Yiying S. Observation of curative effect of Danhong injection on acute exacerbation of chronic obstructive pulmonary disease. $J$ Em Trad Chin Med. 2009;18(8):1232-1233.

108. Liang S, Liangwei F. Clinical experience of Chuankezhi injection in the treatment of chronic obstructive pulmonary disease. Nei Mongol J Traditional Chin Med. 2012;31(5):26-27. doi:10.1186/ 1479-5876-10-26

109. Jin S, Tao S. Observation on curative effect of Tanreqing injection on acute attack of chronic obstructive pulmonary disease. $J \mathrm{Em}$ Trad Chin Med. 2008;17(8):1050.

110. Na T, Qiangmin C. Observation on the Therapeutic Effect of Removing Heat-phlegm Method in the Sequential Treatment of Chronic Obstructive Pulmonary Disease in Acute Exacerbation Period. Guangming J Chin Medi. 2016;22(31):3231-3233.

111. Wei T. Clinical observation on the treatment of acute exacerbation of chronic obstructive pulmonary disease by integrated traditional Chinese and western medicine. Modern Med J China. 2012;14 (3):1016-1017.

112. Rukang T, Xianghua T, Xiaohong L, Yunxia R. Therapeutic effect of Shenmai injection on 38 cases of chronic obstructive pulmonary disease during acute episode. Chin J Modern Drug App. 2009;3(11):134-135.

113. Tulei T. Clinical Study of Reduning Treatment of Acute Exacerbation Phase of Chronic Obstructive Pulmonary Disease. J Heze Med Coll. 2015;27(2):66-68.

114. Aidong W. Clinical observation of Tanreqing injection in treating 72 cases of acute exacerbation of chronic obstructive pulmonary disease. J Med Theory Pract. 2011;24(6):664.

115. Haiyan W. Observation of therapeutic effect of Danzhanxixin injection on acute exacerbation of chronic obstructive pulmonary disease. J New Chin Med. 2010;42(11):10-11.

116. Lixia W, Qingyun L. Therapeutic effect of Tanreqing injection on 50 cases of acute exacerbation of COPD. Chin J Modern Drug App. 2008;2(21):38.

117. Qiu W, Jishan Q. Clinical observation on Tanreqing adjuvant treatment of acute exacerbation of chronic obstructive pulmonary disease. J Modern Med Health. 2013;29(22):3491.

118. Tiejun W, Zhonghua X, Zhenzhong Z. Effects of Shuxuenlng Injection on the Levels of Serum Matrix Metalloproteinase-9 and Tissue Inhibitor of Metalloproteinase-1 in Acute Exacerbated Chronic Obstructive Pulmonary Disease Patients. Chin J Integr Traditional Western Med. 2012;32(2):191-194.

119. Tongbing W. Effects of Tanreqing injection on acute exacerbations of chronic obstructive pulmonary disease. China Pract $J$ Med. 2015;42(017):32-33.

120. Xianghua W, Xiaojun L. Clinical observation on 39 cases of acute exacerbation of chronic obstructive pulmonary disease treated with integrated traditional Chinese and western medicine. Guiding J Traditional Chin Med Pharmacol. 2011;17(7):29-32.

121. Xueqin W. Application of Xuebijing injection in acute exacerbation of chronic obstructive pulmonary disease. Pract Clin J Integr Trad Chin Western Med. 2015;15(5):18-19.

122. Yong W, Chunming S, Yong L. Clinical observation of Shengmai injection in acute exacerbations of chronic obstructive pulmonary disease(COPD). Chin J New Drugs. 2007;16(16):1298-1300.

123. Sizun W, Sining C, Yuan F. Effect of Tanreqing Injection on Cytokines and Lung Function in Patients with acute exacerbation of Chronic Obstructive Pulmonary Disease. J Em Trad Chin Med. 2011;20(9):1402-1403.

124. Yaomin W. Clinical analysis of Reduning injection in the treatment of acute exacerbation of chronic obstructive pulmonary disease. Shenzhen J Integr Traditional Chin Western Med. 2014;24(5):29-30.
125. Yunjun W. Effect of Xuebijing in the treatment of acute exacerbation of chronic obstructive pulmonary disease and its mechanism. Jilin Med J. 2012;33(10):2107-2108.

126. Beishou W, Minqiang $\mathrm{H}$. Clinical study on salvianolate injection in treatment of acute exacerbation of chronic obstructive pulmonary disease. Drugs Clinic. 2015;30(12):1498-1501.

127. Dengxiang W. Clinical observation of 100 cases of acute exacerbation of chronic obstructive pulmonary disease treated with integrated traditional Chinese and western medicine. Contemporary Med. 2015;21(31):157-158.

128. Yi W, Fenghua Q, Lei Z, Yao X, Yimin Q. Effects of Chuankezhi injection with airway humidification on mechanical ventilation function of patients with acute exacerbation of chronic obstructive pulmonary disease. Shanghai J Trad Chin Med. 2017;51(4):57-58.

129. Chunxia X, Xiaohong S. 40 cases of acute exacerbation of COPD treated by Kudiezi injection combined with western medicine. Fujian J Traditional Chin Med. 2010;41(2):17-18.

130. Wei X. Clinical observations on treating respiratory system emergency by Chuankezhi Injection. Chin J Exp Trad Med Formulae. 2012;18(6):252-254.

131. Zhi X, Wei H, Jianbin H, Maofeng X. Observation on curative effect of Tanreqing injection on patients with acute exacerbation of chronic obstructive pulmonary. J Em Trad Chin Med. 2020;29 (2):300-302.

132. Chenxi X, Xiaoxing L. Effects of Acupoint Injection of Astragali Radix Injection on Immune and Respiratory Function of Patients with Sequential Mechanical Ventilation in Acute Exacerbation of Chronic Obstructive Pulmonary Disease. Chin J Information Traditional Chin Med. 2018;25(8):17-21.

133. Yonghong X, Faguang J, Tonggang L, Enqing F, Yani S. Clinical observation of Tanreqing injection in the treatment of acute exacerbation of chronic obstructive pulmonary. J Em Trad Chin Med. 2005;14(4):291-292.

134. Sugiong X, Yaping G, Xi X. Influence of astragalus injection on serum cytokines and lung function in acute exacerbation of chronic obstructive pulmonary disease. Modern Chin Doctor. 2013;51(9):43-45.

135. Weijun X, Xiaojun S, Minzhi D, Xiaowen X, Changyong Z. Clinical observation of atomized inhalation of Tanreqing injection in treating acute exacerbation of chronic obstructive pulmonary disease. J Em Trad Chin Med. 2017;5(26):162-164.

136. Jiewu Y, Min Z. Application of Danhong injection in emergency treatment of patients with Acuet Exacerbation Chronic Obstructive Pulmonary Disease. J Em Trad Chin Med. 2012;21(7):1150.

137. Ruifang Y, Xiue S, Caiyan W, Xiaofeng M. Clinical observation of Danhong injection in the treatment of 42 cases of acute exacerbation of chronic obstructive pulmonary disease in the elderly. Health Vocational Educ. 2010;28(5):139.

138. Weizhong Y, Lanke Z, Zhongtian D, Yuelian L, Yiyun L. Effect analysis of Tanreqing injection in adjuvant treatment of 120 elderly patients with AECOPD. Guangxi Med J. 2014;36 (12):1804-1805.

139. Xiuhong Y. Tanreqing Injection in Adjuvant Treatment of 26 Cases of Acute COPD. Shandong Med J. 2006;46(14):39.

140. Ling Y, Xuchu H. Effect of Xuebijing injection on plasma D-dimer and fibrinogen in patients with AECOPD. $J$ Em Trad Chin Med. 2010;19(10):1674-1675.

141. Shihua Y, Yabo Z, Jijun Y. Application Significance Reduning Injection in the Treatment of Acute Exacerbation of Chronic Obstructive Pulmonary Disease. J North Pharm. 2016;13(6):18-19.

142. Libo Y. Tanreqing injection in treating 32 cases of acute exacerbation of chronic obstructive pulmonary. Jiangxi J Trad Chin Med. 2009;40(10):27-28.

143. Changxiu Y. Clinical study on the adjuvant treatment of chronic obstructive pulmonary disease with Shenmai injection. Zhonghua Yangsheng Baojian. 2020;39(1):40-42. 
144. Chimei Z. Observation of curative effect of astragalus injection on acute exacerbation of chronic obstructive pulmonary disease. Yunnan J Traditional Chin Med Materia Medica. 2014;35(10):54.

145. Li Z. Efficacy of integrated traditional Chinese and western medicine in treating acute exacerbation of chronic obstructive pulmonary disease in the elderly. Chin Foreign Med Res. 2013;11(24):26-27.

146. Liyun Z, Lili L, Wei W. Clinical Observation on Atomized Zhichuanling Injection for the Treatment of 60 Cases with Acute Exacerbation of Chronic Obstructive Pulmonary Disease. World Chin Med. 2017;12(7):1562-1565.

147. Qiang Z. Clinical Analysis of Qingkailing Combined Medication Treatment of Elderly Patients with COPD Infection. Chin J Basic Med Traditional Chin Med. 2015;21(7):841-843.

148. Qiong Z. Randomized controlled study in parallel with acute exacerbation chronic obstructive pulmonary disease of Danhong injection combined with western medicine. J Pract Traditional Chin Internal Med. 2014;28(2):113-114.

149. Wenqian Z. Qingkailing aerosol inhalation treated 36 cases of acute exacerbation of chronic obstructive pulmonary disease. Jilin Med J. 2010;31(9):1215.

150. Xiaohua Z. Efficacy analysis of Honghua injection combined with routine treatment for 47 cases of chronic obstructive pulmonary disease in aggravation stage. China Rural Health. 2016;80(2):7879.

151. Xin Z, Yan W, Aixiang G. Influence of Xiyanping injections therapy on inflammation factors and lung function of old patients with acute exacerbation chronic obstructive pulmonary disease. Clin Med China. 2014;30(9):932-935.
152. Ying Z, Tingqian L, Gang W, et al. Randomized Controlled Trial of Tanreqing Injection in Treatment of Acute Exacerbation of Chronic Obstructive Pulmonary Disease(Syndrome of Retention of Phlegm-Heat in the Lung). Chin J Evid Based Med. 2004;4 (5):300-305.

153. Yuanhua Z, Yunfei G. Curative effect of Kudiezi injection on acute phase of chronic obstructive pulmonary disease. Yunnan $J$ Traditional Chin Med Materia Medica. 2015;36(4):43-44.

154. Zhenhuan Z, Weili J, Yan J, Zhongguo S, Hai D. Clinical Observation of Shuanghuanglian Injection in the Treatment of Acute Exacerbation of Chronic Obstructive Pulmonary Disease. China Pharm. 2016;27(29):4096-4098.

155. Yalil Z, Baoyon H. Effects of Shenfu injection on the patients with acute exacerbation of chronic obstruction pulmonary disease. Inte J Geriatrics. 2017;38(2):63-66.

156. Aizhu Z. Therapeutic effect of Xuesaitong injection on acute exacerbation of chronic obstructive pulmonary disease. J Chin Pract Diagnosis Therapy. 2013;27(7):696-698.

157. Jianguo Z. Clinical effect of Reduning injection on acute exacerbation of chronic obstructive pulmonary disease. Pract J Cardiac Cerebral Pneumal Vasc Dis. 2014;22(11):83-84.

158. Zhong Z, Chuanxiu W. Clinical observation of tanreqing injection in treatment of acute exacerbation of COPD. J Em Trad Chin Med. 2009;18(1):5-6.

159. Xiqing Z, Jianliang Z, Huimin S. Danhong injection in the treatment of acute exacerbation of chronic obstructive pulmonary disease and its effect on hemorheology. J Em Trad Chin Med. 2010;19(2):197.

\section{Publish your work in this journal}

The International Journal of COPD is an international, peer-reviewed journal of therapeutics and pharmacology focusing on concise rapid reporting of clinical studies and reviews in COPD. Special focus is given to the pathophysiological processes underlying the disease, intervention programs, patient focused education, and self management protocols. This journal is indexed on PubMed Central, MedLine and CAS. The manuscript management system is completely online and includes a very quick and fair peer-review system, which is all easy to use. Visit http://www.dovepress.com/testimonials.php to read real quotes from published authors. 